TRANSACTIONS OF THE

AMERICAN MATHEMATICAL SOCIETY

Volume 362, Number 10, October 2010, Pages 5373-5394

S 0002-9947(2010)05029-9

Article electronically published on May 19, 2010

\title{
CHARACTERIZATIONS OF JACOBIANS OF CURVES WITH AUTOMORPHISMS
}

\author{
ESTEBAN GÓMEZ GONZÁLEZ, JOSÉ M. MUÑOZ PORRAS, \\ FRANCISCO J. PLAZA MARTÍN, AND RUBÍ E. RODRÍGUEZ
}

\begin{abstract}
We obtain a characterization of theta functions of Jacobian varieties of curves with automorphisms among theta functions of principally polarized abelian varieties (p.p.a.v.). We first give a characterization in terms of finite dimensional orbits for a suitable action in the Sato Grassmannian. Secondly, the introduction of formal Baker-Akhiezer functions and formal $\tau$ functions attached to a p.p.a.v. (for the multipuncture case) allows us to characterize, in terms of bilinear identities, those Baker-Akhiezer functions that are Baker-Akhiezer functions of Jacobians of curves with automorphisms. Further, in the case of automorphisms with fixed points, we rewrite the previous result as a hierarchy of partial differential equations for the $\tau$-function of a p.p.a.v. Finally, since Baker-Akhiezer and $\tau$ functions are written in terms of theta functions, these results give rise to characterizations of p.p.a.v. in terms of their theta functions.
\end{abstract}

\section{INTRODUCTION}

The objective of the Schottky problem is to characterize the principally polarized abelian varieties (p.p.a.v.) that are Jacobians of smooth algebraic curves. This problem was solved by Shiota ( $\underline{\underline{S}}]$ ) in the framework of the theory of KP equations and by Krichever ([K3]) in terms of the existence of trisecant lines to their Kummer varieties. The analogous problem for Prym varieties was studied and solved by Shiota ([Sh2]) and by Grushevsky and Krichever ([GK] $)$.

The Schottky problem for Prym varieties is also related to the characterization of Jacobians of algebraic curves that admit a non-trivial involution. The moduli space of curves with non-trivial automorphisms was studied in a previous paper (GMP), and the points of the Sato Grassmannian defined by those curves were characterized there. Moreover, an explicit set of algebraic equations defining the moduli space of curves with automorphisms as a subscheme of the Sato Grassmannian was obtained.

The present paper aims at obtaining characterizations of theta functions of Jacobian varieties of curves with automorphisms. More precisely, our first result consists of identifying those points of the Sato Grassmannian coming from curves with an automorphism. This characterization, inspired by that of Mulase for points coming

Received by the editors September 22, 2008.

2010 Mathematics Subject Classification. Primary 14H42, 14H37; Secondary 37K10, 14K25.

Key words and phrases. Principally polarized abelian varieties, Jacobian varieties, Schottky problem, curves with automorphisms, theta functions, KP and KdV hierarchies, completely integrable systems.

This work was partially supported by the research contracts MTM2006-07618 of DGI and SA112A07 of JCyL, and Fondecyt grant 1060742.

(C) 2010 American Mathematical Society Reverts to public domain 28 years from publication 
from curves (Mu1]), is stated in terms of finite dimensional orbits of a suitable action in the Sato Grassmannian.

We also offer a second characterization that determines when a theta function of p.p.a.v. is a theta function of the Jacobian variety of a curve with an automorphism. In other words, it is a solution of the Schottky problem for curves with automorphisms in terms of bilinear identities. In order to obtain this result, we generalize, for the multipuncture case, the notions of Baker-Akhiezer (BA) functions and $\tau$ functions. Then, we attach formal BA-functions and a formal $\tau$-function to the theta function of a p.p.a.v. Theorem 5.6 characterizes the BA functions that are BA functions of Jacobians in terms of two bilinear identities. It is worth pointing out that the first of these identities (equation 2.a of Theorem [5.6) is essentially the multicomponent KP hierarchy and establishes the condition for an arbitrary theta function to be the theta function of a Jacobian. The second one (equation 2.b of Theorem (5.6) provides the condition for this Jacobian variety to be the Jacobian variety of a curve with automorphisms.

The techniques of BA-functions and bilinear identities used in this result are inspired by those of [K1, DJKM] and were used in the first part of Shiota's paper ([Sh] ) in order to solve the Schottky problem in terms of the KP hierarchy. Actually, for the multipuncture case we need generalizations of the KP hierarchy as well as of a result of Shiota ( $\underline{\mathrm{Sh}}$, Thm. 6). This is carried out in $\S 2$ and Theorem 5.3 . respectively.

Finally, we write down a hierarchy of partial differential equations for the $\tau$ function of a p.p.a.v. that is satisfied precisely when it is a $\tau$-function of a Jacobian of a curve admitting an automorphism of order $p$ with fixed points. It is shown that our hierarchy contains the KP hierarchy and yields the KdV for $p=2$.

The authors thank the referee for his helpful suggestions concerning the presentation of this paper.

We would like to dedicate this paper to the memory of our friend Sevín Recillas. Professor Recillas, who passed away June 20, 2005, participated in a preliminary version of this paper presented at the Third Iberoamerican Congress on Geometry (Salamanca, 2004).

\section{Preliminary Results}

2.A. Formal group schemes. In this section we establish the notation and recall results from the papers MP1, MP2, GMP.

Throughout this section, we consider a natural number $p$, the trivial $\mathbb{C}((z))$ algebra $V=\prod_{i=1}^{p} \mathbb{C}((z))$, and its subspaces $V^{+}=\prod_{i=1}^{p} \mathbb{C}[[z]]$ and $V^{-}=$ $\prod_{i=1}^{p} z^{-1} \mathbb{C}\left[z^{-1}\right]$. In order to distinguish the position of the entries, subindices will be employed. Namely, we shall write

$$
V=\mathbb{C}\left(\left(z_{1}\right)\right) \times \cdots \times \mathbb{C}\left(\left(z_{p}\right)\right),
$$

where the $\mathbb{C}((z))$-algebra structure, $\mathbb{C}((z)) \rightarrow V$, maps $z$ to $\left(z_{1}, \ldots, z_{p}\right)$.

The formal base curve is $\widehat{C}:=\operatorname{Spf} \mathbb{C}[[z]]$, and the formal spectral cover is $\widehat{C}_{V}:=$ $\operatorname{Spf} V^{+}$. Let $\Gamma_{V}$ be the formal group scheme representing the functor

$$
\begin{aligned}
\{\text { category of formal } \mathbb{C} \text {-schemes\} } & \rightsquigarrow\{\text { category of groups }\}, \\
S & \rightsquigarrow\left(V \hat{\otimes}_{\mathbb{C}} H^{0}\left(S, \mathcal{O}_{S}\right)\right)_{0}^{*},
\end{aligned}
$$


where the subscript 0 denotes the connected component of the identity and the superscript ${ }^{*}$ denotes the invertible elements. Replacing $V$ by $V^{+}$(respectively by $1+V^{-}$) we define the subgroup $\bar{\Gamma}_{V}^{+}$(respectively $\Gamma_{V}^{-}$) and thus obtain the decomposition

$$
\Gamma_{V}=\Gamma_{V}^{-} \times \bar{\Gamma}_{V}^{+}
$$

The formal Jacobian of the formal spectral cover is the formal group scheme $\mathcal{J}\left(\widehat{C}_{V}\right):=\Gamma_{V}^{-}$. A straightforward calculation shows that $\mathcal{J}\left(\widehat{C}_{V}\right)$ is the formal spectrum of the ring

$$
\mathcal{O}\left(\mathcal{J}\left(\widehat{C}_{V}\right)\right)=\mathbb{C}\left\{\left\{t_{1}^{(1)}, t_{2}^{(1)}, \ldots\right\}\right\} \widehat{\otimes} \cdots \widehat{\otimes} \mathbb{C}\left\{\left\{t_{1}^{(p)}, t_{2}^{(p)}, \ldots\right\}\right\},
$$

where the $t_{i}^{(j)}$ are indeterminates and $\mathbb{C}\left\{\left\{t_{1}, t_{2}, \ldots\right\}\right\}$ denotes the inverse limit $\varliminf_{n} \mathbb{C}\left[\left[t_{1}, \ldots, t_{n}\right]\right]$.

Replacing $V, V^{+}$and $V^{-}$by $\mathbb{C}((z)), \mathbb{C}[[z]]$ and $z^{-1} \mathbb{C}\left[z^{-1}\right]$, respectively, in the previous constructions, one obtains formal schemes $\Gamma, \bar{\Gamma}^{+}$and $\mathcal{J}(\widehat{C}):=\Gamma^{-}$. It is straightforward that the canonical morphism $\mathbb{C}((z)) \hookrightarrow V$ gives rise to $\Gamma \hookrightarrow \Gamma_{V}$ and that the trace and the norm yield corresponding morphisms $\Gamma_{V} \rightarrow \Gamma$.

Recall that the Abel map $\phi_{V}: \widehat{C}_{V} \longrightarrow \mathcal{J}\left(\widehat{C}_{V}\right)$ is the morphism corresponding to the $\widehat{C}_{V}$-valued point of $\Gamma_{V}$ associated to the $p$-tuple of series

$$
\left(\left(1-\frac{\bar{z}_{1}}{z_{1}}\right)^{-1}, \ldots,\left(1-\frac{\bar{z}_{p}}{z_{p}}\right)^{-1}\right)
$$

where $\widehat{C}_{V} \simeq \operatorname{Spf}\left(\mathbb{C}\left[\left[\bar{z}_{1}\right]\right] \times \cdots \times \mathbb{C}\left[\left[\bar{z}_{p}\right]\right]\right)$.

2.B. Infinite Grassmannians. Sato's infinite Grassmannian ([SS]), the set of solutions of the KP hierarchy, was applied to the study of line bundles on curves by Mulase ( $\mathrm{Mu} 1)$ and by Segal and Wilson ( $\underline{\mathrm{SW}}]$ ). The analogues in the case of higher rank are the multicomponent KP hierarchy (e.g. $\mathrm{KvdL}$ ) and the Grassmannian of $\mathbb{C}((z))^{\oplus n}$ (e.g. $[\mathrm{AB}, \overline{\mathrm{LM}}]$ ). Nevertheless, let us rewrite these objects from a scheme-theoretical point of view.

The infinite Grassmannian $\operatorname{Gr}(V)$ of the pair $\left(V, V^{+}\right)$is a $\mathbb{C}$-scheme, not of finite type, whose set of rational points is given as follows:

$$
\left\{\begin{array}{c}
\text { subspaces } U \subset V \text { such that } U \rightarrow V / V^{+} \\
\text {has a finite dimensional kernel and cokernel }
\end{array}\right\} .
$$

This scheme is equipped with the determinant bundle $\operatorname{Det}_{V}$, given by the determinant of the complex of $\mathcal{O}_{\operatorname{Gr}(V)}$-modules

$$
\mathcal{L} \longrightarrow V / V^{+} \hat{\otimes}_{\mathbb{C}} \mathcal{O}_{\operatorname{Gr}(V)},
$$

where $\mathcal{L}$ is the universal submodule of $\operatorname{Gr}(V)$ and the morphism is the natural projection. The connected components of the Grassmannian are indexed by the Euler-Poincare characteristic of the complex. The connected component of index $m$ will be denoted by $\operatorname{Gr}^{m}(V)$.

The group $\Gamma_{V}$ acts by homotheties on $V$, and this action gives rise to a natural action on $\operatorname{Gr}(V)$,

$$
\Gamma_{V} \times \operatorname{Gr}(V) \longrightarrow \operatorname{Gr}(V) .
$$

Furthermore, this action preserves the characteristic and the determinant bundle.

Henceforth, we shall restrict ourselves to $\operatorname{Gr}^{0}(V)$, which will be denoted by $\operatorname{Gr}(V)$ for the sake of simplicity. 
These facts allow us to introduce $\tau$-functions and Baker-Akhiezer functions of points of $\operatorname{Gr}(V)$ (BA-functions for short). Let us recall the definition and some properties of these functions (see [MP2, Section 3]).

The determinant of the morphism $\mathcal{L} \rightarrow V / V^{+} \hat{\otimes}_{\mathbb{C}} \mathcal{O}_{\mathrm{Gr}(V)}$ gives rise to a canonical global section

$$
\Omega_{+} \in H^{0}\left(\operatorname{Gr}(V), \operatorname{Det}_{V}^{*}\right) .
$$

The $\tau$-function $\tau_{U}(t)$ for $U \in \operatorname{Gr}(V)$ is a function on $\mathcal{J}\left(\widehat{C}_{V}\right)$, introduced as a suitable trivialization of the function $g \mapsto \Omega_{+}(g U)$ for $g \in \mathcal{J}\left(\widehat{C}_{V}\right)$,

$$
\tau_{U}(g)=\frac{\Omega_{+}(g U)}{g \delta_{U}}
$$

where $\delta_{U}$ is a non-zero element in the fibre of $\operatorname{Det}_{V}^{*}$ over $U \in \operatorname{Gr}(V)$.

Let $t$ be the set of variables $\left(t^{(1)}, \ldots, t^{(p)}\right)\left(\right.$ where $t^{(j)}=\left(t_{1}^{(j)}, t_{2}^{(j)}, \ldots\right)$ ) and let $z$. denote $\left(z_{1}, \ldots, z_{p}\right)$. For $1 \leq u, v \leq p$, let $\left[z_{v}\right]:=\left(z_{v}, \frac{z_{v}^{2}}{2}, \frac{z_{v}^{3}}{3}, \ldots\right), t+\left[z_{v}\right]:=$ $\left(t^{(1)}, \ldots, t^{(v)}+\left[z_{v}\right], \ldots, t^{(p)}\right), U_{u u}=U$ and, if $u \neq v, U_{u v}:=\left(1, \ldots, z_{u}, \ldots, z_{v}^{-1}, \ldots, 1\right)$. $U$.

Following MP2 and Ma, we consider the $u$-th Baker-Akhiezer function of a point $U \in \operatorname{Gr}(V)$ as the $V$-valued function defined by

$$
\psi_{u, U}(z, t):=\left(\epsilon_{u v} \exp \left(-\sum_{i \geq 1} \frac{t_{i}^{(v)}}{z_{v}^{i}}\right) \frac{\tau_{U_{u v}}\left(t+\left[z_{v}\right]\right)}{\tau_{U}(t)}\right)_{v=1, \ldots, p},
$$

where $\epsilon_{u v}$ is equal to -1 for $u>v$ and 1 otherwise.

The main property of these Baker-Akhiezer functions is that they can be understood as generating functions for $U$ as a subspace of $V$, as we recall next.

Theorem 2.3 (MP2, Thm. 3.6). Let $U \in \operatorname{Gr}(V)$. Then

$$
\psi_{u, U}\left(z_{.}, t\right)=\left(1, \ldots, z_{u}, \ldots, 1\right) \cdot \sum_{i>0}\left(\psi_{u, U}^{(i, 1)}\left(z_{1}\right), \ldots, \psi_{u, U}^{(i, p)}\left(z_{p}\right)\right) p_{u i, U}(t)
$$

where

$$
\left\{\left(\psi_{u, U}^{(i, 1)}\left(z_{1}\right), \ldots, \psi_{u, U}^{(i, p)}\left(z_{p}\right)\right) \mid i>0,1 \leq u \leq p\right\}
$$

is a basis of $U$ and $p_{u i, U}(t)$ are functions in $t$.

Consider the pairing

$$
\begin{aligned}
V \times V & \longrightarrow \mathbb{C} \\
(a, b) & \longmapsto \operatorname{Res}_{z=0} \operatorname{Tr}(a, b) d z
\end{aligned}
$$

where $\operatorname{Tr}: V \longrightarrow \mathbb{C}((z))$ is the trace map. Let $U^{\perp}$ denote the orthogonal complement of a subspace $U$ with respect to this metric. Since the pairing is nondegenerate and the characteristic of $U^{\perp}$ is 0 , there is an involution of $\operatorname{Gr}(V)$ mapping every point $U$ to its orthogonal complement $U^{\perp}$.

Finally, the adjoint Baker-Akhiezer functions of $U$ are defined by

$$
\psi_{u, U}^{*}\left(z_{.}, t\right):=\psi_{u, U^{\perp}}\left(z_{.},-t\right),
$$

and a $p$-tuple of formal functions $\psi_{u}(z, t)$ consists of the BA-functions of a point of $\operatorname{Gr}(V)$ if and only if the $(1, \ldots, 1)$-KP hierarchy

$$
\operatorname{Res}_{z=0} \operatorname{Tr}\left(\frac{\psi_{u}\left(z_{.}, t\right)}{\left(1, \ldots, z_{u}, \ldots, 1\right)} \cdot \frac{\psi_{v}^{*}(z, s)}{\left(1, \ldots, z_{v}, \ldots, 1\right)}\right) d z=0
$$


holds for all $1 \leq v, u \leq p$ and all $t, s$ (for notation and results see [MP2, §3]). This hierarchy is essentially equivalent to the $p$-multicomponent KP hierarchy ( $\mathrm{KvdL}$ ). To see this equivalence, it suffices to repeat the above construction for the $\tau$ functions of the points $\left(z_{1}^{\alpha_{1}}, \ldots, z_{p}^{\alpha_{p}}\right) U$ with $\left(\alpha_{1}, \ldots, \alpha_{p}\right) \in \mathbb{Z}^{p}$ and $\sum_{i} \alpha_{i}=0$.

2.C. Invariant subspaces. The automorphism $\sigma: V \rightarrow V$ of order $p$ defined by $\sigma\left(z_{i}\right)=z_{i+1}$ for $i<p$ and $\sigma\left(z_{p}\right)=z_{1}$ induces an automorphism of $\operatorname{Gr}(V)$ that preserves the determinant line bundle; it will also be denoted by $\sigma$. If $\operatorname{Gr}(V)^{\sigma}$ denotes the set of points in $\operatorname{Gr}(V)$ fixed under the action of $\sigma$, then it is known that $\operatorname{Gr}(V)^{\sigma}$ is a closed subscheme.

The automorphism $\sigma$ also gives rise to an automorphism of $\mathcal{J}\left(\widehat{C}_{V}\right)$ whose expression in terms of its ring of functions is $\sigma^{*}\left(t_{j}^{(i)}\right)=t_{j}^{(i-1)}$ for $i>1$ and $\sigma^{*}\left(t_{j}^{(1)}\right)=t_{j}^{(p)}$. Thus, the corresponding relations are as follows:

$$
\tau_{\sigma(U)}(t)=\tau_{U}\left(\sigma^{*}(t)\right)
$$

(up to a constant) and

$$
\begin{aligned}
\psi_{u, \sigma(U)}\left(z_{\boldsymbol{.}}, t\right) & =\left(\psi_{u+1, U}^{(2)}\left(z_{1}, \sigma^{*}(t)\right), \psi_{u+1, U}^{(3)}\left(z_{2}, \sigma^{*}(t)\right), \ldots, \psi_{u+1, U}^{(1)}\left(z_{p}, \sigma^{*}(t)\right)\right) \\
& =\sigma^{-1}\left(\psi_{u+1, U}\left(z_{\mathbf{.}}, \sigma^{*}(t)\right)\right),
\end{aligned}
$$

where the action of $\sigma$ in the last term is the action on $V$-valued functions.

A point $U \in \operatorname{Gr}(V)$ lies in $\operatorname{Gr}(V)^{\sigma}$ if and only if its BA-functions satisfy the following identities:

$$
\operatorname{Res}_{z=0} \operatorname{Tr}\left(\frac{\psi_{u, \sigma(U)}\left(z_{.}, t\right)}{\left(1, \ldots, z_{u}, \ldots, 1\right)} \cdot \frac{\psi_{v, U}^{*}\left(z_{\bullet}, s\right)}{\left(1, \ldots, z_{v}, \ldots, 1\right)}\right) d z=0
$$

for all $u, v \in\{1, \ldots, p\}$.

\section{The Jacobian CASE}

3.A. Geometrical meaning of "formal" objects. This section aims at exploring the relation between $\tau$-functions and theta functions of Jacobians. In particular, $\tau$-functions attached to a Riemann surface with marked points will be defined following the works of Dubrovin, Fay, Krichever, Shiota and Adler-Shiota-van Moerbecke ([D, F1, K1, K2, Sh, ASvM]).

Throughout this section, $C$ will be an integral complete curve of genus $g$ over $\mathbb{C}$, and $J_{g-1}(C)$ will denote the scheme parametrizing invertible sheaves of degree $g-1$. For the sake of clarity, we shall assume $C$ to be smooth, although most of the results established here hold in greater generality.

Let us fix data $\left(C, \bar{x}, t_{\bar{x}}\right)$, where $\bar{x}=\left\{x_{1}, \ldots, x_{p}\right\}$ are $p$ pairwise distinct points of $C$ and $t_{\bar{x}}$ is a collection of formal parameters $\left\{t_{x_{1}}, \ldots, t_{x_{p}}\right\}$ giving the corresponding isomorphisms $t_{x_{j}}: \widehat{\mathcal{O}}_{C, x_{j}} \stackrel{\sim}{\rightarrow} \mathbb{C}\left[\left[z_{j}\right]\right]$.

Proposition 3.1. For each invertible sheaf $L \in J_{g-1}(C)$, there is a canonical morphism $\gamma: \mathcal{J}\left(\widehat{C}_{V}\right) \rightarrow J_{g-1}(C)$ such that the diagram

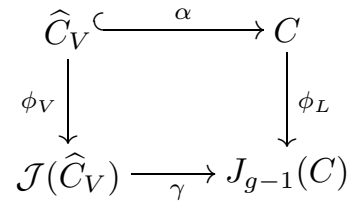


is commutative. Here $\phi_{V}$ is the Abel map (subsection 2.A) and $\phi_{L}$ sends a point $x^{\prime} \in C$ to $L\left(p \cdot x^{\prime}-\bar{x}\right)$.

Proof. The data $\left(C, \bar{x}, t_{\bar{x}}\right)$ gives rise to a canonical morphism:

$$
\mathcal{O}_{C} \hookrightarrow \widehat{\mathcal{O}}_{C, \bar{x}} \stackrel{\sim}{\longrightarrow} V^{+} \text {. }
$$

Then we define $\alpha$ to be the induced morphism between the corresponding schemes.

Because the pair $\left(\mathcal{J}\left(\widehat{C}_{V}\right), \phi_{V}\right)$ satisfies the Albanese property for $\widehat{C}_{V}$ ([MP1), the composition $\widehat{C}_{V} \rightarrow J_{g-1}(C)$ factors through $\phi_{V}$, thus defining $\gamma$.

Let $J_{g-1}^{\infty}(C, \bar{x})$ be the scheme parametrizing pairs $(L, \phi)$, where $L \in J_{g-1}(C)$ and $\phi: \widehat{L}_{\bar{x}} \stackrel{\sim}{\rightarrow} \widehat{\mathcal{O}}_{C, \bar{x}}(\underline{\mathrm{Mu}}, \underline{\mathrm{SW}})$ ). It carries a canonical action of $\bar{\Gamma}_{V}^{+}$, since this group acts by homotheties on the trivialization of $L$. Summing up, there is an exact sequence of group schemes,

$$
0 \rightarrow \bar{\Gamma}_{V}^{+} \rightarrow J_{g-1}^{\infty}(C, \bar{x}) \rightarrow J_{g-1}(C) \rightarrow 0 .
$$

This sequence also has a formal counterpart,

$$
0 \rightarrow \bar{\Gamma}_{V}^{+} \rightarrow \Gamma_{V} \rightarrow \mathcal{J}\left(\widehat{C}_{V}\right) \rightarrow 0 .
$$

Since this latter sequence splits, $\Gamma_{V} \simeq \mathcal{J}\left(\widehat{C}_{V}\right) \times \bar{\Gamma}_{V}^{+}$, and every element $g \in \Gamma_{V}$ can be written as $\left(g_{-}, g_{+}\right)$with $g_{-} \in \mathcal{J}\left(\widehat{C}_{V}\right), g_{+} \in \bar{\Gamma}_{V}^{+}$and $g=g_{-} \cdot g_{+}$.

Proposition 3.2. Let $(L, \phi)$ be a point in $J_{g-1}^{\infty}(C, \bar{x})$. Then, the Abel maps $\phi_{V}$ and $\phi_{L}$ have canonical lifts to $\Gamma_{V}$ and $J_{g-1}^{\infty}(C, \bar{x})$, respectively, and the map $\gamma$ has a lift

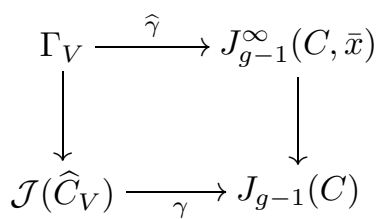

compatible with those of $\phi_{V}$ and $\phi_{L}$.

Proof. Let $E(u, v)$ denote the prime form of $C$ as a holomorphic section of the line bundle associated with the diagonal divisor of $C \times C$. Thus the meromorphic function $\frac{E\left(x, x_{i}\right)}{E\left(x, x^{\prime}\right)}$ has a zero at $x=x_{i}$ and a pole at $x=x^{\prime}$. Moreover, if $z_{i}$ is a coordinate at $x_{i}$ such that $z_{i}\left(x_{i}\right)=0$ and $\bar{z}_{i}:=z_{i}\left(x^{\prime}\right)$, then the expansion of this function at $x_{i}$ is the following series:

$$
t_{x_{i}}\left(\frac{E\left(x, x_{i}\right)}{E\left(x, x^{\prime}\right)}\right) \in\left(1-\frac{\bar{z}_{i}}{z_{i}}\right)^{-1} \cdot\left(1+z_{i} \mathbb{C}\left[\left[z_{i}\right]\right]\right) .
$$

Therefore, the $p$-tuple consisting of the expansions of $\prod_{i=1}^{p} \frac{E\left(x, x_{i}\right)}{E\left(x, x^{\prime}\right)}$ at $x_{1}, \ldots, x_{p}$ corresponds to a morphism $\widehat{C}_{V} \rightarrow \Gamma_{V}$, which lifts the Abel morphism $\phi_{V}$ defined by the $p$-tuple (2.1).

To define the lift of $\phi_{L}$, it suffices to observe that if the line bundle $L$ carries a formal trivialization $\phi: \widehat{L}_{\bar{x}} \stackrel{\sim}{\rightarrow} \widehat{\mathcal{O}}_{C, \bar{x}}$, then $L\left(p \cdot x^{\prime}-\bar{x}\right)$ is canonically endowed with the trivialization given by

$$
\phi \cdot \prod_{i=1}^{p} \frac{E\left(x, x_{i}\right)}{E\left(x, x^{\prime}\right)}
$$


Finally, the lift of $\gamma$ is defined by

$$
\begin{aligned}
\widehat{\gamma}: \Gamma_{V} & \longrightarrow J_{g-1}^{\infty}(C, \bar{x}), \\
g & \longmapsto\left(L \otimes L_{g_{-}}, \phi \cdot g\right),
\end{aligned}
$$

where $L_{g_{-}}$is given as follows: let $D_{i}$ be a small disk around $x_{i}$ such that $z_{i}$ defines a coordinate in $D_{i}$; then $L_{g_{-}}$consists of gluing the trivial bundles on $C-\bar{x}$ and on $\stackrel{\circ}{D}_{1}, \ldots, \stackrel{\circ}{D}_{p}$ by the transition functions $\left(g_{-}\right)_{1}, \ldots,\left(g_{-}\right)_{p}$ (see [SW], Remark 6.8 and Sh, Lemma 4).

Since $\left(C, \bar{x}, t_{\bar{x}}\right)$ has been fixed, the Krichever map is defined as follows:

$$
\begin{aligned}
\mathrm{Kr}: J_{g-1}^{\infty}(C, \bar{x}) & \rightarrow \operatorname{Gr}(V), \\
(L, \phi) & \mapsto\left(t_{\bar{x}} \circ \phi\right)\left(H^{0}(C-\bar{x}, L)\right) .
\end{aligned}
$$

Thus, we obtain the following

Theorem 3.3. For $(L, \phi) \in J_{g-1}^{\infty}(C, \bar{x})$, let $U=\operatorname{Kr}(L, \phi) \in \operatorname{Gr}(V)$.

Then the composition

$$
\Gamma_{V} \stackrel{\widehat{\gamma}}{\longrightarrow} J_{g-1}^{\infty}(C, \bar{x}) \stackrel{\mathrm{Kr}}{\hookrightarrow} \operatorname{Gr}(V)
$$

coincides with the morphism $\mu_{U}: \Gamma_{V} \cong \Gamma_{V} \times\{U\} \rightarrow \operatorname{Gr}(V)$ mapping $g$ to $g \cdot U$.

Proof. We need to check that $(\mathrm{Kr} \circ \widehat{\gamma})(g)=g \cdot U$, but this is an easy consequence of the definition of $\widehat{\gamma}$.

In particular, we have obtained morphisms

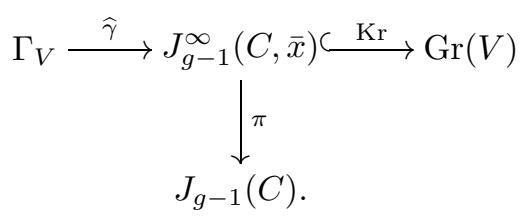

As a straightforward consequence of the determinantal construction of the theta divisor in $J_{g-1}(C)$ and of the determinant bundle on $\operatorname{Gr}(V)$, it follows that

$$
\mathrm{Kr}^{*} \operatorname{Det}_{V}^{*} \simeq \pi^{*} \mathcal{O}_{J_{g-1}}(\Theta) .
$$

In particular, the $\tau$-function of the point $\operatorname{Kr}(L, \phi)$ almost coincides with the theta function at $L$. We clarify this issue in the following subsection.

3.B. $\tau$-function and Baker-Akhiezer functions. The relations just described between the determinant bundle and the theta line bundle induce an explicit relation between $\tau$-functions and theta functions, which we now study.

Let us choose a symplectic basis $\left\{\alpha_{1}, \ldots, \alpha_{g}, \beta_{1}, \ldots, \beta_{g}\right\}$ for $H_{1}(C, \mathbb{Z})$, and let $\left\{\omega_{1}, \ldots, \omega_{g}\right\}$ denote the corresponding canonical basis of holomorphic 1-forms on $C$; that is,

$$
\int_{\alpha_{i}} \omega_{j}=\delta_{i j} \quad \text { and } \quad \int_{\beta_{i}} \omega_{j}=\Omega_{i j}
$$

where $\Omega=\left(\Omega_{i j}\right)$ is the period matrix for $C$. Then the Jacobian variety of $C$, as a complex torus, is defined by

$$
J(C):=\mathbb{C}^{g} / \mathbb{Z}^{g}+\Omega \mathbb{Z}^{g} .
$$


Since $J(C)$ parametrizes the 0 degree line bundles on $C$, the image of $\xi \in \mathbb{C}^{g}$ by the natural quotient map $\mathbb{C}^{g} \rightarrow J(C)$ will be considered as a line bundle of degree 0 and denoted by $L_{\xi}$.

We also recall that the Riemann theta function associated with $\Omega$ is the quasiperiodic function on $\mathbb{C}^{g}$ (the universal cover of $J(C)$ ) given by

$$
\theta(z)=\sum_{m \in \mathbb{Z}^{g}} \exp \left(2 \pi i m^{t} z+\pi i m^{t} \Omega m\right) .
$$

By the Riemann-Kempf Theorem (we follow [F1], Theorem 1.1), there is a theta characteristic $\mathcal{O}_{C}(\Delta) \in J_{g-1}(C)$ inducing an identification of $J(C)$ and $J_{g-1}(C)$ such that the zero divisor of $\theta$ corresponds to the theta divisor $\Theta \subset J_{g-1}(C)$. In particular, with an element $\xi \in \mathbb{C}^{g}$ we associate the line bundle $L_{\xi}(\Delta) \in J_{g-1}(C)$.

Given these data, there is a canonical map

$$
\begin{aligned}
H^{0}\left(C, \Omega_{C}\right) & \longrightarrow \Omega_{\widehat{C}_{V}} \simeq \mathbb{C}\left[\left[z_{1}\right]\right] d z_{1} \times \cdots \times \mathbb{C}\left[\left[z_{p}\right]\right] d z_{p}, \\
\omega_{i} & \mapsto\left(\ldots,\left(a_{i 1}^{(j)}+a_{i 2}^{(j)} z_{j}+a_{i 3}^{(j)} z_{j}^{2}+\ldots\right) d z_{j}, \ldots\right)
\end{aligned}
$$

that maps each $\omega_{i}$ to its local expansions at the points $x_{1}, \ldots, x_{p}$. Since we have chosen bases on both sides, for each $j \in\{1, \ldots, p\}$ we have a $g \times \infty$ matrix $A^{(j)}$ over $\mathbb{C}$, associated with the map $H^{0}\left(C, \Omega_{C}\right) \rightarrow \mathbb{C}\left[\left[z_{j}\right]\right] d z_{j}$. Moreover, $\operatorname{rank} A^{(j)}=g$ for each $j \in\{1, \ldots, p\}$.

Remark 3.4. The transpose of the above map is related to the map induced by Proposition 3.1 at the level of tangent spaces.

For each $j \in\{1, \ldots, p\}$ and each natural number $n$, let $\eta_{n}^{(j)}$ denote the normalized meromorphic 1-form on $C$ with a unique pole of order $n+1$ at $x_{j}$ of the form $d\left(z_{j}^{-(n+1)}\right)+O(1)$ and such that

$$
\int^{x} \eta_{n}^{(j)}=z_{j}^{-n}+O\left(z_{j}\right) \quad \text { at } x=x_{j} .
$$

Observe that the vector of $\beta$-periods of $\eta_{k}^{(k)}$ is precisely $-2 \pi \sqrt{-1}$ times the $j$-th column of $A^{(k)}$.

We also consider the complex numbers $q_{n m}^{(j)}$ defined by the following identities:

$$
\int^{x} \eta_{n}^{(j)}=z_{j}^{-n}-2 \sum_{m=1}^{\infty} q_{n m}^{(j)} \frac{z_{j}^{m}}{m} \quad \text { at } x=x_{j} .
$$

If $t$ is the $p$-tuple $\left(t^{(1)}, \ldots, t^{(p)}\right)$, where each $t^{(j)}$ is a family of variables $\left(t_{1}^{(j)}, t_{2}^{(j)}\right.$, $\ldots)$, then we define the quadratic form

$$
Q(t)=\sum_{n, m \geq 1} q_{n m}^{(1)} t_{n}^{(1)} t_{m}^{(1)}+\cdots+\sum_{n, m \geq 1} q_{n m}^{(p)} t_{n}^{(p)} t_{m}^{(p)}
$$

and the point $A(t)$ of $J(C)$ with values in $\mathbb{C}\left\{\left\{t^{(1)}, \ldots, t^{(p)}\right\}\right\}$ given by

$$
A(t)=A^{(1)} t^{(1)}+\cdots+A^{(p)} t^{(p)} .
$$

We are now ready to introduce the classical Baker-Akhiezer functions and to compare them to those given in $\S 2$. Let us recall Krichever's approach to the multipuncture case, in particular, his Theorem 2.1 in $\underline{\mathrm{K} 2}$. Let us fix a base point $x_{0} \in C$, and let $x \mapsto \int_{x_{0}}^{x} \omega$ denote the Abel map with base point $x_{0}$ and $\omega=$ 
$\left(\omega_{1}, \ldots, w_{g}\right)$. For an effective divisor $D=y_{1}+\cdots+y_{g+p-1}$ of degree $g+p-1$ and for each $1 \leq u \leq p$, let $W\left(D_{u}\right) \in \mathbb{C}^{g}$ be a representative of

$$
\mathcal{O}_{C}\left(\sum_{i} y_{i}-\sum_{i \neq u} x_{i}-\Delta-x_{0}\right) \in J(C) .
$$

For generic $D$ and parameters $t=\left(t^{(1)}, \ldots, t^{(p)}\right)\left(\right.$ with $\left.t^{(k)}=\left(t_{1}^{(k)}, t_{2}^{(k)}, \ldots\right)\right)$, the classical Baker-Akhiezer functions are the functions on $x \in C$ defined by

$$
\exp \left(\sum_{k, i} \int_{x_{0}}^{x} t_{i}^{(k)} \eta_{i}^{(k)}\right) \frac{\theta\left(\int_{x_{0}}^{x} \omega-W\left(D_{u}\right)+A(t)\right)}{\theta\left(\int_{x_{0}}^{x} \omega-W\left(D_{u}\right)\right)} \quad 1 \leq u \leq p .
$$

These functions form a basis of the vector space consisting of the functions $f(x)$ on $C$ such that

- $f$ is meromorphic on $C-\bar{x}$, and $\operatorname{div}(f)+D \geq 0$ on $C-\bar{x}$;

- for each $k, f(x)$ has an essential singularity at $x=x_{k}$ such that $\exp \left(-\sum_{i} t_{i}^{(k)} z_{k}^{-i}\right) f\left(z_{k}\right)$ is holomorphic (recall that $z_{k}$ is a formal parameter at $\left.x_{k}\right)$.

Now let us set a representative $\xi \in \mathbb{C}^{g}$ of $\mathcal{O}_{C}(D-\Delta-\bar{x}) \in J(C)$. Note that, for each function given by (3.5), the vector of its germs at the points $x_{1}, \ldots, x_{p}$ belongs to $V=\mathbb{C}\left(\left(z_{1}\right)\right) \times \cdots \times \mathbb{C}\left(\left(z_{p}\right)\right)$. From the first condition it follows that if we allow the parameters $t$ to vary, these vectors generate a subspace $U_{D} \subset V$ isomorphic to $H^{0}\left(C-\bar{x}, \mathcal{O}_{C}(D)\right)$. In particular, this isomorphism yields a formal trivialization, $\phi_{\xi}$, of the line bundle $L_{\xi}(\Delta)=\mathcal{O}_{C}(D-\bar{x})$ such that

$$
U_{D}=z_{\cdot} \cdot U_{\xi}, \quad \text { where } U_{\xi}:=\left(t_{\bar{x}} \circ \phi_{\xi}\right)\left(H^{0}\left(C-\bar{x}, L_{\xi}(\Delta)\right)\right) .
$$

Therefore, using these arguments and recalling Theorem 2.3, we conclude that there are two ways of constructing generating functions for the subspace $U_{\xi}$. The first one uses the BA-functions of $U_{\xi}$ as a point of $\operatorname{Gr}(V)$ (see $\S 2$ ), while the second one is based on the classical BA-functions of the data $\left(C, \bar{x}, t_{\bar{x}},\left\{\alpha_{1}, \ldots, \beta_{g}\right\}\right.$ ) (see equation (3.5)).

Since the aim of this paper is a characterization of Jacobians among p.p.a.v., we introduce the following definition, which is well behaved for the case of abelian varieties (see $\S 5$ ).

Definition 3.6. For all $u, v$ in $\{1, \ldots, p\}$, let $\xi_{u v} \in \mathbb{C}^{g}$ denote a representative of $\mathcal{O}_{C}\left(x_{u}-x_{v}\right)=\int_{x_{v}}^{x_{u}} \omega \in J(C)$ such that $\xi_{u v}+\xi_{v w}=\xi_{u w}$ and $\xi_{u u}=0$.

The $u$-th BA-function of

$$
\left(J(C), \Omega,\left\{A^{(1)}, \ldots, A^{(p)}\right\},\left\{Q^{(1)}, \ldots, Q^{(p)}\right\},\left\{\xi_{u v}\right\}_{1 \leq u, v \leq p}, \xi\right)
$$

is the $V$-valued function, given as follows:

$$
\bar{\psi}_{u, \xi}\left(z_{.}, t\right):=\left(\epsilon_{u v} \exp \left(-\sum_{i \geq 1} \frac{t_{i}^{(v)}}{z_{v}^{i}}\right) \frac{\tau\left(\xi+\xi_{u v}, t+\left[z_{v}\right]\right)}{\tau(\xi, t)}\right)_{v=1, \ldots, p},
$$

where $\epsilon_{u v}$ is equal to -1 for $u>v$ and 1 otherwise, and $\tau(\xi, t)$ is the $\tau$-function

$$
\tau(\xi, t):=\exp (Q(t)) \theta(A(t)+\xi) .
$$

Furthermore, the $u$-th adjoint BA-function is given by

$$
\bar{\psi}_{u, \xi}^{*}\left(z_{*}, t\right):=\bar{\psi}_{u,-\xi}\left(z_{*},-t\right) .
$$


Now, comparing $\bar{\psi}_{u, \xi}(z, t)$ defined by (3.7) with the function defined by (3.5), we have that they coincide up to a normalization at $t=0$. Furthermore, recalling the results of $\S 2$, we may conclude that these functions yield a third generating system of $U_{\xi}$. A fundamental consequence is that the bilinear identity holds for the BA-functions of $\S 2$ if and only if it holds for the functions defined by (3.7). These arguments are central for the proof of Theorem 5.3 .

\section{Characterization as Finite dimensional orbits}

In this section we characterize the points of the Sato Grassmannian that arise from geometric data over an algebraic curve with automorphisms via the Krichever construction. We prove that these points are those whose orbit under the action of $\Gamma_{V}$ is finite dimensional (up to the action of $\bar{\Gamma}_{V}^{+}$). In the paper [GMP], a similar result has been established for a certain subgroup of $\Gamma_{V}$. This type of characterization dates back to the approach of Mulase (Mu1]).

Let us denote by $\overline{\operatorname{Pic}}(C)$ the moduli space of rank 1 torsion free sheaves on $C$.

Consider a curve $C$, an automorphism $\sigma$ of $C$ of order $p$, a divisor $\bar{x}$ composed by $p$ pairwise distinct smooth points $x_{1}, \ldots, x_{p}$ in $C$, and $L \in \overline{\operatorname{Pic}}(C)$.

We say that $(C, \sigma, \bar{x}, L)$ is maximal if the following condition holds (see [SW, p. 38]):

- Let $\left(C^{\prime}, \sigma^{\prime}, \bar{x}^{\prime}, L^{\prime}\right)$ be another such quadruple, and let us assume that there exists a birational morphism $\psi: C^{\prime} \rightarrow C$ such that $\sigma \circ \psi=\psi \circ \sigma^{\prime}, \psi\left(\bar{x}^{\prime}\right)=\bar{x}$ and $\psi_{*} L^{\prime} \stackrel{\sim}{\rightarrow} L$; then $\psi$ is an isomorphism.

Definition 4.1. Let $\underline{\overline{\mathrm{Pic}}}^{\infty}(p)$ denote the contravariant functor from the category of $\mathbb{C}$-schemes to the category of sets defined by

$$
S \rightsquigarrow\left\{\left(C, \sigma_{C}, \bar{x}, t_{\bar{x}}, L, \phi_{\bar{x}}\right)\right\},
$$

where

(1) $p_{C}: C \rightarrow S$ is a proper and flat morphism whose fibres are geometrically reduced curves.

(2) $\sigma_{C}: C \rightarrow C$ is an order $p$ automorphism (over $S$ ).

(3) $\bar{x}=\left\{x_{1}, \ldots, x_{p}\right\}$, where $x_{i}: S \rightarrow C$ are disjoint smooth sections of $p_{S}$ $(i=1, \ldots, p)$ such that $\sigma_{C}\left(x_{i}\right)=x_{i+1}$ for $i<p$ and $\sigma_{C}\left(x_{p}\right)=x_{1}$. Also, for every closed point $s \in S$ and each irreducible component of $C_{s}$, there is at least one $i$ such that $x_{i}(s)$ lies on that component.

(4) $t_{\bar{x}}$ is an equivariant formal parameter along $\bar{x}(S)$, that is, an equivariant isomorphism of $\mathcal{O}_{S}$-modules $t_{\bar{x}}: \widehat{\mathcal{O}}_{C, \bar{x}(S)} \stackrel{\sim}{\rightarrow} \widehat{V}_{S}^{+}$.

(5) $L \in \overline{\operatorname{Pic}}(C)$ satisfies the condition that $\left(C_{s}, \sigma_{C_{s}}, \bar{x}(s),\left.L\right|_{C_{s}}\right)$ is maximal for all closed point $s \in S$.

(6) $\phi_{\bar{x}}$ is a formal trivialization of $L$ along $\bar{x}(S)$, that is, an isomorphism $\phi_{\bar{x}}$ : $\widehat{L}_{\bar{x}(S)} \simeq \widehat{\mathcal{O}}_{C, \bar{x}(S)}$.

(7) $\left(C, \sigma_{C}, \bar{x}, t_{\bar{x}}, L, \phi_{\bar{x}}\right)$ and $\left(C^{\prime}, \sigma_{C^{\prime}}, \bar{x}^{\prime}, t_{\bar{x}^{\prime}}, L^{\prime}, \phi_{\bar{x}^{\prime}}\right)$ are said to be equivalent when there is an isomorphism of $S$-schemes $C \stackrel{\sim}{\rightarrow} C^{\prime}$ compatible with all the data.

The Krichever morphism for the functor $\overline{\mathrm{Pic}}^{\infty}(p)$ is the morphism of functors

$$
\mathrm{Kr}: \underline{\overline{\operatorname{Pic}}}^{\infty}(p) \longrightarrow \underline{\operatorname{Gr}(V)}
$$


that sends the $S$-valued point $\left(C, \sigma_{C}, \bar{x}, t_{\bar{x}}, L, \phi_{\bar{x}}\right)$ to the following submodule of $\widehat{V}_{S}:=V \widehat{\otimes} \mathcal{O}_{S}$ :

$$
\left(t_{\bar{x}} \circ \phi_{\bar{x}}\right)\left(\underset{m}{\lim }\left(p_{C}\right)_{*} L(m \cdot \bar{x})\right) \subset \widehat{V}_{S}
$$

Theorem 4.2. The functor $\overline{\mathrm{Pic}}^{\infty}(p)$ is representable by a subscheme $\overline{\mathrm{Pic}}^{\infty}(p)$ of $\operatorname{Gr}(V)$.

Proof. Consider the morphism from $\overline{\mathrm{Pic}}^{\infty}(p)$ to $\operatorname{Gr}(V) \times \operatorname{Gr}(V)$ which sends the $S$-valued point $\left(C, \sigma_{C}, \bar{x}, t_{\bar{x}}, L, \phi_{\bar{x}}\right)$ to the following pair of submodules:

$$
\left(t_{\bar{x}}\left(\underset{m}{\lim }\left(p_{C}\right)_{*} \mathcal{O}_{C}(m \cdot \bar{x})\right),\left(t_{\bar{x}} \circ \phi_{\bar{x}}\right)\left(\underset{m}{\lim }\left(p_{C}\right)_{*} L(m \cdot \bar{x})\right)\right) \in \operatorname{Gr}(V) \times \operatorname{Gr}(V),
$$

where $p_{C}: C \times S \rightarrow C$ is the projection.

From the inverse construction of the Krichever map ([K1, SW]), this map is injective and its image is contained in the set $Z$ of those pairs $(\mathcal{A}, \mathcal{L})$ in $\operatorname{Gr}(V) \times$ $\operatorname{Gr}(V)$ such that

$$
\mathcal{O}_{S} \subset \mathcal{A} \quad, \quad \mathcal{A} \cdot \mathcal{A} \subseteq \mathcal{A} \quad, \quad \mathcal{A} \cdot \mathcal{L} \subseteq \mathcal{L} \quad, \quad \sigma(\mathcal{A})=\mathcal{A}
$$

Let us examine the maximality condition. For $(\mathcal{A}, \mathcal{L})$ satisfying the above conditions, let $A_{\mathcal{L}}$ denote the stabilizer of $\mathcal{L}$,

$$
A_{\mathcal{L}}:=\left\{v \in \widehat{V}_{S} \text { such that } v \cdot \mathcal{L} \subseteq \mathcal{L}\right\},
$$

and let $\left(C^{\prime}, \sigma_{C^{\prime}}, \bar{x}^{\prime}, t_{\bar{x}^{\prime}}, L^{\prime}, \phi_{\bar{x}^{\prime}}\right)$ be the geometric data defined by the pair $\left(A_{\mathcal{L}}, \mathcal{L}\right)$. Then the inclusion $\mathcal{A} \subseteq A_{\mathcal{L}}$ gives rise to an equivariant morphism of $S$-schemes $\psi: C^{\prime} \rightarrow C$ such that $\psi\left(\bar{x}^{\prime}\right)=\bar{x}$ and $\psi_{*} L^{\prime} \simeq L$. The maximality condition tells us that $\psi_{s}$ is an isomorphism for every closed point $s \in S$. That is, $A_{\mathcal{L}}$ is a finite $\mathcal{A}$ module such that $\mathcal{A}_{s}=\left(A_{\mathcal{L}}\right)_{s}$ for all $s$. Therefore, we have that $\mathcal{A}=A_{\mathcal{L}}$. Summing up, we are interested in the subset $Z_{0}$ of $Z$ consisting of those pairs $(\mathcal{A}, \mathcal{L})$ such that $\mathcal{A}=\mathcal{A}_{\mathcal{L}}$.

From the proof of Theorem 6.5 of [MP1 we know that the condition $\mathcal{A}_{\mathcal{L}} \subseteq \mathcal{A}$ is closed, and hence so is $Z_{0}$. The Krichever construction implies that $Z_{0}$ represents $\overline{\mathrm{Pic}}^{\infty}(p)$. Finally, $\left.p_{2}\right|_{Z_{0}}: Z_{0} \hookrightarrow \operatorname{Gr}(V)$ is a closed immersion (where $p_{2}$ denotes the projection onto the second factor), and the theorem is proved.

Let $\mathcal{C} \rightarrow \overline{\operatorname{Pic}}^{\infty}(p)$ be the universal curve. Then the subscheme of $\overline{\operatorname{Pic}}^{\infty}(p)$, consisting of the points $s$ such that $\mathcal{C}_{s}$ is a smooth curve, is an open subscheme. Therefore, the subfunctor $\underline{\mathrm{Pic}}^{\infty}(p)$ of $\underline{\mathrm{Pic}}^{\infty}(p)$, consisting of those data $\left(C, \sigma_{C}, \bar{x}, t_{\bar{x}}, L, \phi_{\bar{x}}\right)$ such that the fibres $C_{s}$ are smooth curves for all closed points $s \in S$, is representable by an open subscheme $\operatorname{Pic}^{\infty}(p)$ of $\overline{\operatorname{Pic}}^{\infty}(p)$.

Let us consider the action of $\Gamma_{V}$ on $\operatorname{Gr}(V)^{p}:=\operatorname{Gr}(V) \times \cdots \times \operatorname{Gr}(V)$ :

$$
\begin{aligned}
\mu^{p}: \Gamma_{V} \times \operatorname{Gr}(V)^{p} & \longrightarrow \operatorname{Gr}(V)^{p}, \\
\left(g,\left(U_{1}, \ldots, U_{p}\right)\right) & \mapsto\left(g U_{1}, \ldots, g U_{p}\right),
\end{aligned}
$$

and the closed immersion

$$
\begin{aligned}
\operatorname{Gr}(V) & \hookrightarrow \operatorname{Gr}(V)^{p} \\
U & \mapsto U_{\sigma}:=\left(U, \sigma(U), \ldots, \sigma^{p-1}(U)\right) .
\end{aligned}
$$


Let $\Gamma_{V}\left(U_{\sigma}\right)$ denote the orbit of $U_{\sigma}$ under the action of $\Gamma_{V}$, that is, the schematic image of

$$
\mu_{U_{\sigma}}: \Gamma_{V} \times\left\{U_{\sigma}\right\} \longrightarrow \operatorname{Gr}(V)^{p} .
$$

Theorem 4.3. Let $U$ be a closed point of $\operatorname{Gr}(V)$. Then the following conditions are equivalent:

(1) $\operatorname{dim}_{\mathbb{C}} T_{U_{\sigma}}\left(\Gamma_{V}\left(U_{\sigma}\right) / \bar{\Gamma}_{V}^{+}\right)<\infty$, and

(2) there exists $\left(C, \sigma_{C}, \bar{x}, t_{\bar{x}}, L, \phi_{\bar{x}}\right) \in \overline{\mathrm{Pic}}^{\infty}(p)$ such that its image by the Krichever morphism is $U$.

Proof. Arguments similar to those given in the proof of GMP, Theorem 4.12] show that $\Gamma_{V}\left(U_{\sigma}\right) / \bar{\Gamma}_{V}^{+}$is a formal scheme. Following [Mu1, we obtain a natural isomorphism

$$
T_{U_{\sigma}}\left(\Gamma_{V}\left(U_{\sigma}\right) / \bar{\Gamma}_{V}^{+}\right) \simeq T_{1} \Gamma_{V} /\left(\operatorname{Ker} d \mu_{U_{\sigma}}+T_{1} \bar{\Gamma}_{V}^{+}\right),
$$

where $d \mu_{U_{\sigma}}^{p}$ is the map induced by $\mu_{U_{\sigma}}^{p}$ on the respective tangent spaces.

Since $d \mu_{U_{\sigma}}^{p}$ is explicitly given by

$$
\begin{aligned}
V \simeq T_{1} \Gamma_{V} & \stackrel{d \mu_{U_{\sigma}}^{p}}{\longrightarrow} T_{U_{\sigma}} \operatorname{Gr}(V)^{p} \simeq \prod_{i=0}^{p-1} \operatorname{Hom}\left(\sigma^{i}(U), V / \sigma^{i}(U)\right), \\
g & \longmapsto\left(\ldots, \sigma^{i}(U) \hookrightarrow V \stackrel{g}{\rightarrow} V \rightarrow V / \sigma^{i}(U), \ldots\right),
\end{aligned}
$$

it follows that

$$
A_{U}:=\operatorname{Ker} d \mu_{U_{\sigma}}=\cap_{i=0}^{p-1}\left\{g \in V \mid \sigma^{i}(g) U \subseteq U\right\}
$$

is the maximal $\mathbb{C}$-subalgebra of $V$ carrying an action of $\sigma$ and such that $U$ is an $A_{U}$-module.

The claim now follows from the following well-known facts. First, the equivalence between the following three conditions: $\operatorname{dim}_{\mathbb{C}} T_{U_{\sigma}}\left(\Gamma_{V}\left(U_{\sigma}\right) / \bar{\Gamma}_{V}^{+}\right)<\infty, A_{U} \in \operatorname{Gr}(V)$, and $A_{U}=\operatorname{Kr}\left(C, \sigma_{C}, \bar{x}, t_{\bar{x}}\right)$ for some $\left(C, \sigma_{C}, \bar{x}, t_{\bar{x}}\right)$.

Second, that if any one of the three previous conditions holds, then there exists a pair $\left(L, \phi_{\bar{x}}\right)$ such that $\left(C, \sigma_{C}, \bar{x}, t_{\bar{x}}, L, \phi_{\bar{x}}\right) \in \underline{\overline{\operatorname{Pic}}}^{\infty}(p)$ and $U=\operatorname{Kr}\left(C, \sigma_{C}, \bar{x}, t_{\bar{x}}, L, \phi_{\bar{x}}\right)$.

Remark 4.4. If the conditions of Theorem 4.3 hold, then the constructions in $₫ 3 . \mathrm{A}$ imply the existence of a canonical isomorphism,

$$
\Gamma_{V}(U) / \bar{\Gamma}_{V}^{+} \simeq J_{g-1}(C)_{L},
$$

where $J_{g-1}(C) \hat{L}$ denotes the formal completion of $J_{g-1}(C)$ at $L$.

\section{Characterization in terms of Bilinear equations}

In this section we give the conditions that a theta function of a p.p.a.v. must satisfy in order to be the theta function of the Jacobian of a smooth irreducible projective curve with non-trivial automorphism.

Inspired by the results of $\S 3$, we introduce the notion of BA- and $\tau$ functions for the case of any p.p.a.v.

Let $\Omega$ be a point in the Siegel upper half-space such that the principally polarized abelian variety $X_{\Omega}:=\mathbb{C}^{g} /\left(\mathbb{Z}^{g}+\Omega \mathbb{Z}^{g}\right)$ is irreducible. Let $\theta(z)=\theta(z, \Omega)$ denote the Riemann theta function of $X_{\Omega}$. 
For a natural number $p$, consider the following data:

- $A^{(j)}=\left(a_{1}^{(j)}, a_{2}^{(j)}, \ldots\right) \in\left(\mathbb{C}^{g}\right)^{\infty}$, a $g \times \infty$-matrix of rank $g$ for each $j=1, \ldots, p$

- $Q^{(j)}\left(t^{(j)}\right)=\sum_{i, k=1}^{\infty} q_{i k}^{(j)} t_{i}^{(j)} t_{k}^{(j)}$, where $q_{i k}^{(j)} \in \mathbb{C}$, a quadratic form for each $j=1, \ldots, p$;

- $\left\{\xi_{u v} \in \mathbb{C}^{g}\right\}_{1 \leq u, v \leq p}$ such that $\xi_{u v}+\xi_{v w}=\xi_{u w}$ and $\xi_{u u}=0$.

Definition 5.2. To each $\xi \in \mathbb{C}^{g}$ and data

$$
\left(X_{\Omega}, \Omega,\left\{A^{(1)}, \ldots, A^{(p)}\right\},\left\{Q^{(1)}, \ldots, Q^{(p)}\right\},\left\{\xi_{u v}\right\}_{1 \leq u, v \leq p}\right)
$$

satisfying (5.1), we associate its $u$-th BA-function as the $V$-valued function given as follows:

$$
\psi_{u, \xi}(z, t):=\left(\epsilon_{u v} \exp \left(-\sum_{i \geq 1} \frac{t_{i}^{(v)}}{z_{v}^{i}}\right) \frac{\tau\left(\xi+\xi_{u v}, t+\left[z_{v}\right]\right)}{\tau(\xi, t)}\right)_{v=1, \ldots, p},
$$

where $\tau(\xi, t)$ is the $\tau$-function defined by

$$
\tau(\xi, t):=\exp (Q(t)) \theta(A(t)+\xi) .
$$

Furthermore, its $u$-th adjoint BA-function is defined by

$$
\psi_{u, \xi}^{*}(z, t):=\psi_{u,-\xi}\left(z_{.},-t\right) .
$$

We begin with the proof of a generalization of some of the results of Mulase ([Mu1]) and Shiota ([Sh] in terms of the Sato Grassmannian.

Theorem 5.3. Let $X_{\Omega}$ be an irreducible p.p.a.v. of dimension $g$ and let $p$ be a natural number.

Then the following conditions are equivalent:

(1) There exists a triple $\left(C, \bar{x}, t_{\bar{x}}\right)$, where $C$ is a projective irreducible smooth curve of genus $g, \bar{x}=\left(x_{1}, \ldots, x_{p}\right)$ is a p-tuple of distinct points in $C$ and $t_{\bar{x}}=\left(t_{x_{1}}, \ldots, t_{x_{p}}\right)$ is a p-tuple of local parameters at the corresponding $x_{j}$, such that as a p.p.a.v. $X_{\Omega}$ is isomorphic to the Jacobian of $C$.

(2) There exist data $\left\{\left\{A^{(j)}\right\},\left\{Q^{(j)}\right\},\left\{\xi_{u v}\right\}\right\}$ such as in (5.1), where for every $\xi \in \mathbb{C}^{g}$ the corresponding $\tau$-function $\tau(\xi, t)$ is a $\tau$-function of the $(1, . p ., 1)$ KP hierarchy (2.4).

Proof. (1) $\Rightarrow(2)$ This follows from subsection 3.B.

$(2) \Rightarrow(1)$ We denote

$$
A(t)=\sum_{j=1}^{p} A^{(j)} t^{(j)} \quad \text { and } \quad Q(t)=\sum_{j=1}^{p} \sum_{i, k \geq 1} q_{i k}^{(j)} t_{i}^{(j)} t_{k}^{(j)} .
$$

Since $\tau(\xi, t)$ is a $\tau$-function of the $(1, . p ., 1)$-KP hierarchy for every $\xi \in \mathbb{C}^{g}$, it follows that $\tau(\xi, t)$ defines a point $U_{\xi} \in \operatorname{Gr}(V)$ such that $\tau(\xi, t)=\tau_{U_{\xi}}(t)$ (up to a constant). From Theorem 2.3 we have that

$$
U_{\xi}=\left\langle\frac{1}{\left(1, \ldots, z_{u}, \ldots, 1\right)}\left(p_{i}\left(\widetilde{\partial}_{t}\right) \psi_{u, \xi}^{(1)}\left(z_{1}, t\right)_{\mid t=0}, \ldots, p_{i}\left(\widetilde{\partial}_{t}\right) \psi_{u, \xi}^{(p)}\left(z_{p}, t\right)_{\mid t=0}\right)\right\rangle_{\substack{i \leq 0 \\ 1 \leq u \leq p}} .
$$

Therefore, we have obtained a morphism

$$
\begin{aligned}
\varphi: \mathbb{C}^{g} & \longrightarrow \operatorname{Gr}(V), \\
\xi & \longmapsto U_{\xi} .
\end{aligned}
$$


We claim that this morphism induces an injection

$$
X \hookrightarrow \operatorname{Gr}(V) .
$$

Indeed, given $\xi_{1}$ and $\xi_{2}$ in $\mathbb{C}^{g}$, the condition $U_{\xi_{1}}=U_{\xi_{2}}$ is equivalent to $\tau\left(\xi_{1}, t\right)=$ $\tau\left(\xi_{2}, t\right)$ for all $t$ (up to a constant), which is in turn equivalent to $\theta\left(A(t)+\xi_{1}\right)=$ $\theta\left(A(t)+\xi_{2}\right)$ for all $t$ (up to a constant), and therefore equivalent to $\xi_{1}-\xi_{2} \in \mathbb{Z}^{g}+\Omega \mathbb{Z}^{g}$, since $X$ is principally polarized.

Now, the function $A$ may be interpreted as a surjective linear map

$$
\mathbb{C}^{\infty} \times \stackrel{p}{\cdots} \times \mathbb{C}^{\infty} \stackrel{A}{\longrightarrow} \mathbb{C}^{g},
$$

and, with the identifications $\mathbb{C}^{\infty} \times \stackrel{p}{\cdots} \times \mathbb{C}^{\infty}=T_{1} \Gamma_{V}^{-}$and $T_{\xi} X=\mathbb{C}^{g}, A$ corresponds to a surjective morphism of formal group schemes

$$
\Gamma_{V}^{-} \stackrel{A_{\xi}}{\longrightarrow} \hat{X}_{\xi} .
$$

We now claim that the surjective morphism

$$
\begin{aligned}
\mu_{\xi}: \Gamma_{V}^{-} & \longrightarrow \Gamma\left(U_{\xi}\right) / \bar{\Gamma}_{V}^{+}, \\
g & \longmapsto g \cdot U_{\xi}
\end{aligned}
$$

factorizes through $A_{\xi}: \Gamma_{V}^{-} \rightarrow \hat{X}_{\xi}$. Observe that if $s=\left(s^{(1)}, \ldots, s^{(p)}\right) \in \operatorname{Ker} A$, then

$$
\tau_{U_{\xi}}(t+s)=\tau(\xi, t+s)=q_{\xi}(t, s) \exp (Q(t)) \theta(A(t)+\xi)=q_{\xi}(t, s) \tau(\xi, t),
$$

where $q_{\xi}(t, s)$ is an exponential of a linear function in $t$. Generalizing Lemma 3.8 of [SW] to the case of $\Gamma_{V}$, there exists $g \in \bar{\Gamma}_{V}^{+}$(which depends on $s$ ) such that

$$
\tau_{U_{\xi}}(t+s)=q_{\xi}(t, s) \tau(\xi, t)=\tau_{g \cdot U_{\xi}}(t) .
$$

Hence, there is a factorization

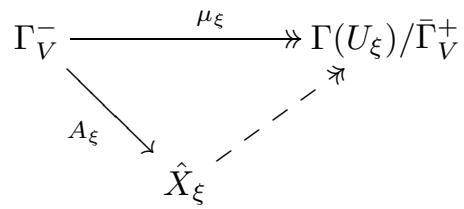

In particular, it follows that $\operatorname{dim} T_{U_{\xi}} \Gamma\left(U_{\xi}\right) / \bar{\Gamma}_{V}^{+}$is finite, and applying the results of [Mu1] one has that there exist data $\left(C_{\xi}, \bar{x}_{\xi}, t_{\xi}, L_{\xi}, \phi_{\xi}\right)$ associated with $U_{\xi}$ under the Krichever map.

We claim that the subset of data $\left(C_{\xi}, \bar{x}_{\xi}, t_{\xi}\right)$ does not depend on $\xi$. Indeed, for $\xi, \xi^{\prime} \in X$ let $s \in \mathbb{C}^{\infty} \times \stackrel{p}{.} \times \mathbb{C}^{\infty}$ be such that $A(s)=\xi^{\prime}-\xi$. We thus have

$$
\begin{aligned}
\tau_{\xi}(t+s) & =\exp (Q(t+s)) \theta(A(t+s)+\xi) \\
& =q_{\xi^{\prime}}(t) \exp (Q(t)) \theta\left(A(t)+\xi^{\prime}\right)=q_{\xi^{\prime}}(t) \tau_{\xi^{\prime}}(t) .
\end{aligned}
$$

A generalization of Lemma 3.8 in [SW] implies that there exists $g_{\xi^{\prime}}(z) \in V$ such that $U_{\xi^{\prime}}=g_{\xi^{\prime}}(z) U_{\xi}$. It follows that $U_{\xi^{\prime}}$ and $U_{\xi}$ have the same stabilizer, and hence $\left(C_{\xi}, \bar{x}_{\xi}, t_{\xi}\right)$ does not depend on $\xi$; it will be denoted by $\left(C, \bar{x}, t_{\bar{x}}\right)$.

The latter fact does have further consequences. It implies that the map (5.4) takes values into $\overline{\operatorname{Pic}}^{\infty}(C, \bar{x})$ (the subscheme of $\operatorname{Gr}(V)$ parameterizing torsion free 
sheaves of rank 1 on $C$ with a formal trivialization along $\bar{x}$ ). Furthermore, it says that the composite map

$$
\begin{aligned}
& X \longleftrightarrow \overline{\operatorname{Pic}}^{\infty}(C, \bar{x}) \longrightarrow \overline{\operatorname{Pic}}(C), \\
& \xi^{\prime} \longmapsto U_{\xi^{\prime}} \longmapsto L_{\xi^{\prime}}
\end{aligned}
$$

takes values in $\operatorname{Pic}^{0}(C) \cdot L_{\xi}$, the orbit of $L_{\xi} \in \overline{\operatorname{Pic}}(C)$ under the action of $\operatorname{Pic}^{0}(C)$. Using the surjectivity of $A$ we can show that the induced map

$$
X \longrightarrow \operatorname{Pic}^{0}(C) \cdot L_{\xi}
$$

is surjective. Since $\left(C, \bar{x}, t_{\bar{x}}, L_{\xi}, \phi_{\xi}\right)$ is maximal (see $\S 4$ ), the action of $\operatorname{Pic}^{0}(C)$ on $\overline{\operatorname{Pic}}(C)$ is free. Accordingly, $\operatorname{Pic}^{0}(C)$ is a quotient of an abelian variety and, therefore, $C$ is a smooth complete curve of genus at most $g$.

To finish the implication $(2) \Rightarrow(1)$, it suffices to show that $X \rightarrow J(C)=\operatorname{Pic}^{0}(C)$ is an isomorphism of p.p.a.v.'s. We do this below. Given $(X, \xi)$ and $(J(C), \xi)$ we consider the $\tau$-functions $\tau_{X}=\tau(\xi, t)$ associated with $X$ as in (5.2) and $\tau_{J}=\tau(\xi, t)$ associated with $J(C)$ as in (3.8). By construction of the data $\left(C_{\xi}, \bar{x}_{\xi}, t_{\xi}, L_{\xi}, \phi_{\xi}\right)$, it follows that $\tau_{X}=\tau_{J}$ (up to a constant), and hence

$$
\theta_{X}(A(t)+\xi)=\exp (q(t)) \theta_{J}\left(A_{J}(t)+\xi\right) \quad \text { (up to a constant) }
$$

where $q(t)$ is a quadratic function. In particular, $\left(\theta_{X}\right)_{0}=\left(\theta_{J}\right)_{0}$ and hence $X$ and $J(C)$ are isomorphic as p.p.a.v., and therefore the curve $C$ is irreducible and of genus $g$.

Remark 5.5. Considering $p=1$ in the previous theorem we obtain the characterization of Jacobian varieties given by Shiota (see [Sh, Theorem 6]).

We shall now apply this result to give a sufficient and necessary condition for a theta function of a p.p.a.v. to be the theta function of a curve with non-trivial automorphisms.

Theorem 5.6 (Characterization). Let $X_{\Omega}$ be an irreducible p.p.a.v. of dimension $g>1$.

Then the following conditions are equivalent:

(1) There exists a projective irreducible smooth curve $C$ of genus $g$ with a non-trivial automorphism $\sigma_{C}: C \rightarrow C$ such that $X_{\Omega}$ is isomorphic as a p.p.a.v. to the Jacobian of $C$.

(2) There exist a prime number $p$ and data $\left\{\left\{A^{(j)}\right\},\left\{Q^{(j)}\right\},\left\{\xi_{u v}\right\}\right\}$ as in (5.1) such that

a) for some $\xi_{0} \in \mathbb{C}^{g}$, the corresponding BA-functions satisfy the $(1, . \stackrel{p}{.}, 1)$ KP hierarchy

$$
\operatorname{Res}\left(\sum_{j=1}^{p} z^{-\delta_{j u}-\delta_{j v}} \psi_{u, \xi_{0}}^{(j)}(z, t) \psi_{v, \xi_{0}}^{*(j)}(z, s)\right) d z=0,
$$

b) there exist $\xi_{1} \in \mathbb{C}^{g}$ (depending on $\xi_{0}$ ) such that

$$
\begin{aligned}
& \operatorname{Res}\left(\sum_{j=1}^{p} z^{-\delta_{j u}-\delta_{j v}} \psi_{v+1, \xi_{0}}^{(j+1)}\left(z, \sigma^{*}(t)\right) \psi_{u, \xi_{1}}^{*(j)}(z, s)\right) d z=0, \\
& \text { where } \sigma^{*}(t):=\left(t^{(p)}, t^{(1)}, t^{(2)}, \ldots, t^{(p-1)}\right) .
\end{aligned}
$$


Proof. (1) $\Rightarrow(2)$ Observe that any curve $C$ with a non-trivial automorphism group admits an automorphism $\sigma_{C}$ of prime order, say $p$, with an orbit $\bar{x}=\left\{x_{1}, \ldots, x_{p}\right\}$ consisting of $p$ different points in $C$. Let $t_{\bar{x}}=\left\{t_{x_{1}}, \ldots, t_{x_{p}}\right\}$ be a collection of local parameters, $t_{x_{j}}$, at each respective $x_{j}$. Now, by Theorem 5.3, there exist $A(t)$ and

$Q(t)$ as in Section 3.B. such that their associated BA-functions $\psi_{u, \xi_{0}}^{(j)}(z, t)$ defined by (3.7) satisfy (2)a) of the statement for each $\xi_{0}$ in $\mathbb{C}^{g}$. That is, there exists a point $U_{\xi_{0}} \in \operatorname{Gr}(V)$ such that $\psi_{u, \xi_{0}}=\psi_{u, U_{\xi_{0}}}$.

Furthermore, the induced embedding $J(C) \hookrightarrow \operatorname{Gr}(V)$ given by (5.4) is compatible with the actions of $\sigma_{C}$ on $J(C)$ and of $\sigma$ on $\operatorname{Gr}(V)$; that is, $\sigma\left(U_{\xi}\right)=U_{\sigma_{C}^{*}} L_{\xi}$. This fact, together with the relation (2.5) applied to the BA-functions $\psi_{u, \xi_{0}}$ for $U_{\xi_{0}}$ and $\psi_{v, \sigma\left(\xi_{0}\right)}$ for $U_{\xi_{1}}=\sigma\left(U_{\xi_{0}}\right)$, prove (2)b).

$(2) \Rightarrow(1)$ We know from Theorem 5.3 that (2)a) implies that there exists a triple $\left(C, \bar{x}, t_{\bar{x}}\right)$ such that $X_{\Omega} \cong J(C)$ as p.p.a.v.'s.

The condition (2)b) implies that $U_{\xi_{1}}=\sigma\left(U_{\xi_{0}}\right)$. In particular, $A_{\xi_{1}}=\sigma\left(A_{\xi_{0}}\right)$, where $A_{\xi}$ denotes the stabilizer of $U_{\xi}$. Hence the orbit $\Gamma_{V}\left(U_{\xi_{0}}, \sigma\left(U_{\xi_{0}}\right) / \bar{\Gamma}_{V}^{+}\right.$is finite dimensional, and it follows from Theorem 4.3 that $\sigma$ induces an automorphism of $A_{\xi_{0}}$. Since $A_{\xi_{0}}=t_{\bar{x}}\left(H^{0}\left(C-\bar{x}, \mathcal{O}_{C}\right)\right)$, because of the Krichever construction, it follows that $C$ admits an automorphism $\sigma_{C}$.

Remark 5.7. Observe that if condition (2) in Theorem 5.3 holds for one $\xi \in \mathbb{C}^{g}$, then it holds for every $\xi$ (see [Sh, Theorem 6]). Therefore, if condition (2)a) in Theorem 5.6 holds for a given $\xi_{0} \in \mathbb{C}^{g}$, then it holds for every $\xi_{0}$. Moreover, under the hypotheses for this theorem, assume further that there are $\xi_{0}$ and $\xi_{1}$ in $\mathbb{C}^{g}$ such that $\xi_{0}-\xi_{1} \in \mathbb{Z}^{g}+\Omega \mathbb{Z}^{g}$ and such that (2)a) and (2)b) are satisfied. Then there exists a line bundle $L$ on $C$ such that $\sigma^{*}(L) \simeq L$.

\section{Characterization in terms of Differential equations (FIXED-POINT CASE)}

Although characterizing Jacobians of curves admitting automorphisms with fixed points is a particular case of the results proved in 5 , we shall now show that in this case we can state a characterization theorem in terms of standard $\tau$-functions for the KP hierarchy.

Since in this case orbits consisting of a single point (counted $p$ times, where $p$ is a prime number) are required, we are forced to replace the previously considered vector space by $V=\mathbb{C}\left(\left(z_{1}\right)\right)$, where its $\mathbb{C}((z))$-algebra structure is given by mapping $z$ to $z_{1}^{p}$, and $\sigma \in \operatorname{Aut}_{\mathbb{C}((z))}(V)$ is given by $\sigma\left(z_{1}\right)=\omega z_{1}$, with $\omega$ a primitive $p$-th root of 1 in $\mathbb{C}$. In this case, we set $V^{+}=\mathbb{C}\left[\left[z_{1}\right]\right]$ and $V^{-}=z_{1}^{-1} \mathbb{C}\left[z_{1}^{-1}\right]$.

The formal base curve in this case is $\widehat{C}=\operatorname{Spf} \mathbb{C}[[z]]$, and the formal spectral cover is $\widehat{C}_{V}:=\operatorname{Spf} V^{+} . \Gamma_{V}$ and $\mathcal{J}\left(\widehat{C}_{V}\right)$ are defined as before, but this case is simpler. Now, $\mathcal{J}\left(\widehat{C}_{V}\right)$ is the formal spectrum of the ring

$$
\mathcal{O}\left(\mathcal{J}\left(\widehat{C}_{V}\right)\right)=\mathbb{C}\left\{\left\{t_{1}, t_{2}, \ldots\right\}\right\} .
$$

For any $\lambda$ in $\mathbb{C}$ and $t=\left(t_{1}, t_{2}, \ldots\right)$, we denote

$$
\lambda t=\left(\lambda t_{1}, \lambda^{2} t_{2}, \ldots\right)
$$

with this notation, the action of $\sigma$ on $\mathcal{J}\left(\widehat{C}_{V}\right)$ in this case is given by $\sigma^{*}(t)=w^{-1} t$. 
The automorphism $\sigma$ induces an automorphism on $\operatorname{Gr}(V)$ (also denoted by $\sigma$ ), and $\operatorname{Gr}(V)^{\sigma}$ will be the set of points of $\operatorname{Gr}(V)$ invariant under the action of $\sigma$, which is a closed subscheme of $\operatorname{Gr}(V)$.

The action of $\sigma$ on $\operatorname{Gr}(V)$ may be described in terms of the $\tau$ and Baker functions as follows. Recall that the $\tau$-function of a point $U \in \operatorname{Gr}(V)$ is given by equation (2.2), and hence it holds that

$$
\tau_{\sigma(U)}(t)=\tau_{U}\left(\omega^{-1} t\right)
$$

(up to a constant). The BA-function of $U$ has the following expression:

$$
\psi_{U}\left(z_{1}, t\right):=\exp \left(-\sum_{i \geq 1} \frac{t_{i}}{z_{1}^{i}}\right) \frac{\tau_{U}\left(t+\left[z_{1}\right]\right)}{\tau_{U}(t)},
$$

and, consequently, one has that

$$
\psi_{\sigma(U)}\left(z_{1}, t\right)=\psi_{U}\left(\omega^{-1} z_{1}, \omega^{-1} t\right)=\sigma^{-1}\left(\psi_{U}\left(z_{1}, \sigma^{*}(t)\right)\right),
$$

where the last expression corresponds to the action of $\sigma$ on $V$; that is, since $\psi_{U}\left(z_{1}, t\right)$ is $V$-valued, $\sigma$ acts on $z_{1}$ and acts trivially on $t$.

It is also known that a point $U \in \operatorname{Gr}(V)$ lies in $\operatorname{Gr}(V)^{\sigma}$ if and only if its BAfunctions satisfy the following identities ([MP2, Theorem 3.11]):

$$
\operatorname{Res}_{z=0} \operatorname{Tr}\left(\frac{1}{z_{1}} \psi_{\sigma(U)}\left(z_{1}, t\right) \psi_{U}^{*}\left(z_{1}, s\right)\right) \frac{d z}{z}=0
$$

or, equivalently, for all $0 \leq k \leq p-1$,

$$
\operatorname{Res}_{z=0} \operatorname{Tr}\left(\frac{1}{z_{1}} \psi_{\sigma^{k}(U)}\left(z_{1}, t\right) \psi_{U}^{*}\left(z_{1}, s\right)\right) \frac{d z}{z}=0 .
$$

Remark 6.3. Using these explicit expressions, it is straightforward to repeat the arguments of $\S 4$ and obtain a characterization of Jacobians of curves admitting an automorphism with fixed points in terms of finite dimensional orbits.

In analogy with Theorem 5.6, we now give a necessary and sufficient condition for a theta function of a p.p.a.v. to be the theta function of the Jacobian of a curve admitting an order $p$ automorphism with fixed points.

Theorem 6.4. Let $X_{\Omega}$ be an irreducible p.p.a.v. of dimension $g$.

Then the following conditions are equivalent:

(1) There exists a quadruple $\left(C, \sigma_{C}, x, t_{x}\right)$, where $C$ is a projective irreducible smooth curve of genus $g, \sigma_{C}$ is an automorphism of order $p$ of $C, x$ is a fixed point of $\sigma_{C}$ in $C$, and $t_{x}$ is a local parameter at $x$, such that $X_{\Omega}$ is isomorphic as a p.p.a.v. to the Jacobian of $C$.

(2) There exists a $g \times \infty$-matrix $A$ of rank $g$ and a symmetric quadratic form $Q(t)$ such that for each $\xi_{0}$ in $\mathbb{C}^{g}$ there exists $\xi_{1}$ in $\mathbb{C}^{g}$ so that the corresponding BA-functions satisfy

a) the KP hierarchy

$$
\operatorname{Res}_{z=0} \psi_{\xi_{0}}(z, t) \psi_{\xi_{0}}^{*}(z, s) \frac{d z}{z^{2}}=0 \text { and }
$$

b) the identity

$$
\operatorname{Res}_{z=0} \psi_{\xi_{0}}\left(\omega^{-1} z, \omega^{-1} t\right) \psi_{\xi_{1}}^{*}(z, s) \frac{d z}{z^{2}}=0
$$

for all $t$ and $s$, where $\omega$ is a primitive $p$-th root of 1 . 
Proof. This is similar to the proof of Theorem 5.6.

We may translate this result into differential equations; indeed, it will suffice to apply the standard arguments in [F1] (see also [MP1]). We know that condition (2)a) in the theorem is equivalent to the KP hierarchy, and, thus, it remains for us to study condition (2)b).

Theorem 6.5. Let $V=\mathbb{C}\left(\left(z_{1}\right)\right)$ and let $U$ be a closed point of $\operatorname{Gr}(V)$.

Then $U$ is a point of $\operatorname{Gr}(V)^{\sigma}$ if and only its $\tau$-function $\tau_{U}$ satisfies the differential equations

$$
\sum_{\substack{\beta_{1}+\beta_{2}-\alpha_{1}-\alpha_{2}=1 \\ \alpha_{1}-\beta_{1} \equiv j}}\left(D_{\lambda_{1}, \alpha_{1}}\left(-\tilde{\partial}_{x}\right) p_{\beta_{1}}\left(\tilde{\partial}_{x}\right) D_{\lambda_{2}, \alpha_{2}}\left(\tilde{\partial}_{s}\right) p_{\beta_{2}}\left(-\tilde{\partial}_{s}\right)\right) \tau_{U}(x) \tau_{U}(s)=0
$$

for all Young diagrams $\lambda_{1}, \lambda_{2}$ and all integers $j \in\{0, \ldots, p-1\}$, where the definition of $D_{\lambda, \alpha}$ is

$$
D_{\lambda, \alpha}\left(\tilde{\partial}_{y}\right)(f(y)):=\sum_{\lambda-\mu=(\alpha)} \chi_{\mu}\left(\tilde{\partial}_{y}\right)(f(y))_{\left.\right|_{y=0}} .
$$

Proof. Let $U$ be a closed point of $\operatorname{Gr}(V)$. Thus, we know that $U \in \operatorname{Gr}(V)^{\sigma}$ if and only if the identities (6.2) are fulfilled. These conditions are equivalent to the vanishing of the constant term of

$$
\sum_{j=1}^{p} \frac{1}{\omega^{j} z_{1}} \psi_{\sigma^{k}(U)}\left(\omega^{j} z_{1}, t\right) \psi_{U}^{*}\left(\omega^{j} z_{1}, s\right)=\sum_{j=1}^{p} \frac{1}{\omega^{j} z_{1}} \psi_{U}\left(\omega^{j-k} z_{1}, \omega^{-k} t\right) \psi_{U}^{*}\left(\omega^{j} z_{1}, s\right)
$$

for each $0 \leq k \leq p-1$.

Let $p_{n}(t)$ be the Schur polynomials and $\widetilde{\partial}_{t^{(j)}}=\left(\partial_{t_{1}^{(j)}}, \frac{1}{2} \partial_{t_{2}^{(j)}}, \frac{1}{3} \partial_{t_{3}^{(j)}}, \ldots\right)$. Recall from [F1] (see also MP1]) that

$$
\psi_{U}\left(z_{1}, t\right)=\exp \left(-\sum_{i \geq 1} \frac{t_{i}}{z_{1}^{i}}\right) \frac{\tau_{U}\left(t+\left[z_{1}\right]\right)}{\tau_{U}(t)}=\left(\sum_{i \geq 0} \frac{p_{i}(-t)}{z_{1}^{i}}\right) \frac{\left(\sum_{i \geq 0} p_{i}\left(\widetilde{\partial}_{t}\right) z_{1}^{i}\right) \tau_{U}(t)}{\tau_{U}(t)}
$$

and that

$$
\psi_{U}^{*}\left(z_{1}, s\right)=\exp \left(\sum_{i \geq 1} \frac{s_{i}}{z_{1}^{i}}\right) \frac{\tau_{U}\left(s-\left[z_{1}\right]\right)}{\tau_{U}(s)}=\left(\sum_{i \geq 0} \frac{p_{i}(s)}{z_{1}^{i}}\right) \frac{\left(\sum_{i \geq 0} p_{i}\left(-\widetilde{\partial}_{s}\right) z_{1}^{i}\right) \tau_{U}(s)}{\tau_{U}(s)} .
$$

Using both expressions above and equation (6.1), the coefficients we are looking for turn out to be

$$
\begin{aligned}
& \sum_{j=1}^{p} \sum_{\beta_{1}+\beta_{2}-\alpha_{1}-\alpha_{2}=1} \omega^{j\left(\beta_{2}+\beta_{1}-\alpha_{1}-\alpha_{2}-1\right)+k\left(\alpha_{1}-\beta_{1}\right)} \\
& \cdot p_{\alpha_{1}}\left(-\omega^{-k} t\right) p_{\beta_{1}}\left(\tilde{\partial}_{\omega^{-k} t}\right) \tau_{U}\left(\omega^{-k} t\right) \cdot p_{\alpha_{2}}(s) p_{\beta_{2}}\left(-\tilde{\partial}_{s}\right) \tau_{U}(s) \\
&= p \sum_{\ell=0}^{p-1}\left(\omega^{k}\right)^{\ell} \sum_{\substack{\beta_{1}+\beta_{2}-\alpha_{1}-\alpha_{2}=1 \\
\alpha_{1}-\beta_{1}=\ell}} p_{\alpha_{1}}\left(-\omega^{-k} t\right) p_{\beta_{1}}\left(\tilde{\partial}_{\omega^{-k}}\right) \tau_{U}\left(\omega^{-k} t\right) \\
& \cdot p_{\alpha_{2}}(s) p_{\beta_{2}}\left(-\tilde{\partial}_{s}\right) \tau_{U}(s) .
\end{aligned}
$$


Now let us note that by substituting $\omega^{-k} t$ by $x$ we have that

$$
\begin{gathered}
\sum_{\substack{\beta_{1}+\beta_{2}-\alpha_{1}-\alpha_{2}=1 \\
\alpha_{1}-\beta_{1} \equiv \ell \\
(\bmod p)}} p_{\alpha_{1}}\left(-\omega^{-k} t\right) p_{\beta_{1}}\left(\tilde{\partial}_{\omega^{-k} t}\right) \tau_{U}\left(\omega^{-k} t\right) p_{\alpha_{2}}(s) p_{\beta_{2}}\left(-\tilde{\partial}_{s}\right) \tau_{U}(s) \\
=\sum_{\substack{\beta_{1}+\beta_{2}-\alpha_{1}-\alpha_{2}=1 \\
\alpha_{1}-\beta_{1} \equiv \ell(\bmod p)}} p_{\alpha_{1}}(-x) p_{\beta_{1}}\left(\tilde{\partial}_{x}\right) \tau_{U}(x) p_{\alpha_{2}}(s) p_{\beta_{2}}\left(-\tilde{\partial}_{s}\right) \tau_{U}(s),
\end{gathered}
$$

and hence the coefficients are

$$
p \sum_{\ell=0}^{p-1}\left(\omega^{k}\right)^{\ell} \sum_{\substack{\beta_{1}+\beta_{2}-\alpha_{1}-\alpha_{2}=1 \\ \alpha_{1}-\beta_{1} \equiv \ell \\(\bmod p)}} p_{\alpha_{1}}(-x) p_{\beta_{1}}\left(\tilde{\partial}_{x}\right) \tau_{U}(x) p_{\alpha_{2}}(s) p_{\beta_{2}}\left(-\tilde{\partial}_{s}\right) \tau_{U}(s) .
$$

Since they must vanish for each $0 \leq k \leq p-1$ (note that the case $k=0$ is precisely the KP hierarchy; see [MP1], we obtain the equivalent conditions

$$
\sum_{\substack{\beta_{1}+\beta_{2}-\alpha_{1}-\alpha_{2}=1 \\ \alpha_{1}-\beta_{1} \equiv \ell}} p_{\alpha_{1}}(-x) p_{\beta_{1}}\left(\tilde{\partial}_{x}\right) \tau_{U}(x) p_{\alpha_{2}}(s) p_{\beta_{2}}\left(-\tilde{\partial}_{s}\right) \tau_{U}(s)=0
$$

for all values of $x$ and $s$, and for all $\ell$ in $\{0, \ldots, p-1\}$.

Since the vanishing of a function $f(x, s)$ (such as the left hand side of (6.7)) for all values of $x$ and $s$ is equivalent to the vanishing of $\chi_{\lambda_{1}}\left(\tilde{\partial}_{x}\right) \chi_{\lambda_{2}}\left(\tilde{\partial}_{s}\right) f(x, s)_{\left.\right|_{x=0, s=0}}$ for all Young diagrams $\lambda_{1}$ and $\lambda_{2}$, a calculation shows that (6.7) is equivalent to (6.6), thus proving the theorem.

Remark 6.8. We now compute the first equations in the previous statement and relate them to the KP equations. Observe that if $E_{j}$ denotes the left hand side of (6.6) for $j \in\{0, \ldots, p-1\}$, then the $\mathrm{KP}$ hierarchy is $E_{0}+\cdots+E_{p-1}=0$. Let us make this explicit.

The first non-trivial equation in the KP hierarchy, which corresponds to the Young diagrams $\lambda_{1}=(1,1,1)$ and $\lambda_{2}=0$, is the celebrated KP equation

$$
\begin{aligned}
\tau_{U}(0) \chi_{(2,2)}\left(\tilde{\partial}_{s}\right) \tau_{U}(s)_{\left.\right|_{s=0}}-p_{1}\left(\tilde{\partial}_{x}\right) \tau_{U}(x)_{\left.\right|_{x=0}} \cdot \chi_{(2,1)}\left(\tilde{\partial}_{s}\right) \tau_{U}(s)_{\left.\right|_{s=0}} \\
+p_{2}\left(\tilde{\partial}_{x}\right) \tau_{U}(x)_{\left.\right|_{x=0}} \chi_{(1,1)}\left(\tilde{\partial}_{s}\right) \tau_{U}(s)_{\left.\right|_{s=0}}=0 .
\end{aligned}
$$

On the other hand, equation (6.6) for the same Young diagrams are

$$
\left\{\begin{array}{l}
\left.p_{1}\left(\tilde{\partial}_{x}\right) \tau_{U}(x)\right|_{\left.\right|_{x=0}} \cdot \chi_{(2,1)}\left(\tilde{\partial}_{s}\right) \tau_{U}(s)_{\left.\right|_{s=0}}=0, \\
\tau_{U}(0) \chi_{(2,2)}\left(\tilde{\partial}_{s}\right) \tau_{U}(s)_{\left.\right|_{s=0}}+p_{2}\left(\tilde{\partial}_{x}\right) \tau_{U}(x)_{\left.\right|_{x=0}} \chi_{(1,1)}\left(\tilde{\partial}_{s}\right) \tau_{U}(s)_{\left.\right|_{s=0}}=0
\end{array}\right.
$$

for $p=2$ and

for $p \neq 2$.

$$
\left\{\begin{array}{l}
p_{1}\left(\tilde{\partial}_{x}\right) \tau_{U}(x)_{\left.\right|_{x=0}} \cdot \chi_{(2,1)}\left(\tilde{\partial}_{s}\right) \tau_{U}(s)_{\left.\right|_{s=0}}=0 \\
\tau_{U}(0) \chi_{(2,2)}\left(\tilde{\partial}_{s}\right) \tau_{U}(s)_{\left.\right|_{s=0}}=0 \\
p_{2}\left(\tilde{\partial}_{x}\right) \tau_{U}(x)_{\left.\right|_{x=0}} \chi_{(1,1)}\left(\tilde{\partial}_{s}\right) \tau_{U}(s)_{\left.\right|_{s=0}}=0
\end{array}\right.
$$

We are now ready to write a hierarchy of partial differential equations that characterizes those $\tau$-functions (and hence the theta functions) associated with Jacobian varieties of curves admitting a non-trivial automorphism with fixed points. 
Theorem 6.9. Let $X_{\Omega}$ be an irreducible p.p.a.v. of dimension $g$.

Then the following conditions are equivalent:

(1) There exists a quadruple $\left(C, \sigma_{C}, x, t_{x}\right)$, where $C$ is a projective irreducible smooth curve of genus $g, \sigma_{C}$ is an automorphism of order $p$ of $C, x$ is a fixed point of $\sigma_{C}$ in $C$, and $t_{x}$ is a local parameter at $x$, such that $X_{\Omega}$ is isomorphic as a p.p.a.v. to the Jacobian of $C$.

(2) There exists a $g \times \infty$-matrix $A$ of rank $g$, a symmetric quadratic form $Q(t)$, and $\xi_{0}$ in $X[2]$ (the subgroup of 2-torsion points in $X$ ) such that

$$
\begin{aligned}
& \sum_{\substack{\beta_{1}+\beta_{2}-\alpha_{1}-\alpha_{2}=1 \\
\alpha_{1}-\beta_{1} \equiv j(\bmod p)}}\left(D_{\lambda_{1}, \alpha_{1}}\left(-\tilde{\partial}_{x}\right) p_{\beta_{1}}\left(\tilde{\partial}_{x}\right) D_{\lambda_{2}, \alpha_{2}}\left(\tilde{\partial}_{s}\right) p_{\beta_{2}}\left(-\tilde{\partial}_{s}\right)\right) \tau\left(\xi_{0}, x\right) \tau\left(\xi_{0}, s\right)=0 \\
& \quad \text { for all } j \text { in }\{0,1, \ldots, p-1\} \text { and all } \lambda_{1}, \lambda_{2} \text {. }
\end{aligned}
$$

Proof. (1) $\Rightarrow(2)$ If (1) holds, then there exists a theta characteristic invariant under $\sigma$. Indeed, for the case $p=2$ this follows from [F2] (see page 87). For $p>2$, note that $\sigma$ acts on $X[2]$ and that $\sigma$ has odd order while $X[2]$ has $2^{2 g}$ points, hence there is a fixed point. This theta characteristic gives rise to $\xi_{0}$ in $X[2]$, and then

$$
U_{\xi_{0}} \in \operatorname{Gr}(V)^{\sigma} .
$$

It follows from Theorem 6.5 that equation (6.10) is satisfied by $\tau_{U_{\xi_{0}}}$.

$(2) \Rightarrow(1)$ It follows from Remark 6.8 that the KP hierarchy is satisfied. Now, Theorem 5.3 implies that there exists a triple $\left(C, x, t_{x}\right)$ such that $X_{\Omega} \simeq J(C)$ as a p.p.a.v. Further, let $U_{\xi_{0}} \in \operatorname{Gr}(V)$ be the point whose $\tau$-function coincides with $\tau\left(\xi_{0}, t\right)$.

Since equation (6.10) holds, Theorem 6.5 implies that the point $U_{\xi_{0}}$ belongs to $\operatorname{Gr}(V)^{\sigma}$. In particular, it follows that $\sigma\left(A_{U_{\xi_{0}}}\right)=A_{U_{\xi_{0}}}$, where $A_{U_{\xi_{0}}}$ denotes the stabilizer of $U_{\xi_{0}}$. Recalling that $A_{U_{\xi_{0}}}=t_{x}\left(H^{0}\left(C-x, \mathcal{O}_{C}\right)\right)$, we deduce that $C$ admits an order $p$ automorphism fixing $x$.

Remark 6.11. Observe that according to the previous theorem in order to know whether a $\tau$-function of a p.p.a.v. is a $\tau$-function of the Jacobian variety of a curve with an automorphism with a fixed point, one has only to check the hierarchy (6.10) a finite number of instances, namely, once for each $\xi_{0} \in X[2]$. Note that, from Remark 6.8 the hierarchy (6.10) implies the KP hierarchy and hence the KP equation.

Example 6.12. As an example we give some more equations from (6.6) for other pairs of Young diagrams. Note that the corresponding equations in the KP hierarchy are all trivial.

(1) Consider the Young diagrams $\lambda_{1}=0$ and $\lambda_{2}=(1)$ and the corresponding $p$ equations given by (6.6).

If $p=2$, both equations are trivial.

However, if $p \neq 2$, then two of the equations (6.6) (the cases $\alpha_{1}-\beta_{1} \equiv 0$ $(\bmod p)$ and $\left.\alpha_{1}-\beta_{1} \equiv-2(\bmod p)\right)$ are equivalent to the equation

$$
\tau_{U}(0) \cdot p_{2}\left(\tilde{\partial}_{x}\right) \tau_{U}(x)_{\left.\right|_{x=0}}=0 \text {. }
$$

Similarly, the consideration of $\lambda_{1}=(1)$ and $\lambda_{2}=0$ in (6.6) yields trivial equations for $p=2$, whereas for $p \neq 2$ we obtain

$$
\tau_{U}(0) \cdot p_{2}\left(-\tilde{\partial}_{x}\right) \tau_{U}(x)_{\mid x=0}=0 .
$$


(2) More generally, for $n \geq 2$ and not divisible by $p$ we obtain

$$
\begin{aligned}
\tau_{U}(0) \cdot p_{n}\left(\tilde{\partial}_{t}\right) \tau_{U}(t)_{\left.\right|_{t=0}} & =0, \\
\tau_{U}(0) \cdot p_{n}\left(-\tilde{\partial}_{t}\right) \tau_{U}(t)_{\left.\right|_{t=0}} & =0
\end{aligned}
$$

by considering (6.6) with $\lambda_{i}=(n-1)$ and $\lambda_{j}=0$.

(3) When $n \geq 2$ is not divisible by $p$, we obtain

$$
\begin{aligned}
p_{1}\left(\tilde{\partial}_{t}\right) \tau_{U}(t)_{\left.\right|_{t=0}} \cdot p_{n+1}\left(\tilde{\partial}_{s}\right) \tau_{U}(s)_{\left.\right|_{s=0}} & =0, \\
p_{1}\left(\tilde{\partial}_{t}\right) \tau_{U}(t)_{\left.\right|_{t=0}} \cdot p_{n+1}\left(-\tilde{\partial}_{s}\right) \tau_{U}(s)_{\left.\right|_{s=0}} & =0
\end{aligned}
$$

by considering $\lambda_{i}=(n-1,1)$ and $\lambda_{j}=0$.

(4) When $n \geq 1$ is not divisible by $p$, the equations become

$$
\begin{aligned}
p_{2}\left(\tilde{\partial}_{t}\right) \tau_{U}(t)_{\left.\right|_{t=0}} \cdot p_{n+2}\left(\tilde{\partial}_{s}\right) \tau_{U}(s)_{\left.\right|_{s=0}} & =0, \\
\left.p_{2}\left(-\tilde{\partial}_{t}\right) \tau_{U}(t)_{\left.\right|_{t=0}} \cdot p_{n+2}\left(-\tilde{\partial}_{s}\right) \tau_{U}(s)\right|_{\left.\right|_{s=0}} & =0
\end{aligned}
$$

for $\lambda_{i}=(n+1,2)$ and $\lambda_{j}=0$.

Remark 6.13. Let us study the relation to the KdV hierarchy. Let us consider an invariant point $U \in \operatorname{Gr}(V)^{\sigma}$. If we impose the condition that $\mathbb{C}\left[z^{-1}\right] \cdot U=$ $\mathbb{C}\left[z_{1}^{-p}\right] \cdot U=U$, then we obtain the $p$-KdV hierarchy. To see this, recall that the $\tau$-function $\tau_{U}$ of $U$ is (up to a constant) the pullback of the global section $\Omega_{+}$to $\Gamma_{V}$ by

$$
\Gamma_{V} \times\{U\} \longrightarrow \operatorname{Gr}(V) .
$$

Since the condition means that $\Gamma^{-} \cdot U=U$, we obtain the following diagram:

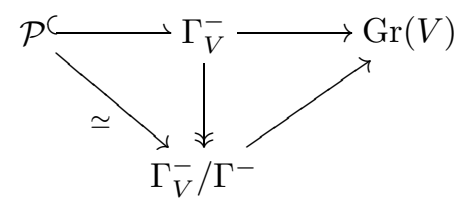

where

$$
\mathcal{P}:=\left\{g \in \mathcal{J}\left(\widehat{C}_{V}\right) \mid \operatorname{Nm}(g)=1\right\}=\left\{\exp \left(\sum t_{i} z_{1}^{-i}\right) \in \Gamma_{V} \mid t_{i}=0 \text { for } i=\dot{p}\right\}
$$

and $\mathrm{Nm}$ is the norm of $V$ as a $\mathbb{C}((z))$-algebra (GMP).

Therefore, for $p=2$ with fixed points, it makes sense to write $\tau_{U}=\tau_{U}\left(t_{1}, t_{3}, \ldots\right)$ as an element of $\mathcal{O}(\mathcal{P})=\mathbb{C}\left\{\left\{t_{1}, t_{3}, \ldots\right\}\right\}$ so that the $\tau$-function only depends on $t_{i}$ with $i$ odd. The resulting hierarchy is the classical KdV hierarchy (see also SW, Proposition 5.11]).

\section{REFERENCES}

[AB] Adams, M. R.; Bergvelt, M. J., "The Krichever map, vector bundles over algebraic curves, and Heisenberg algebras", Comm. Math. Phys. 154 (1993), no. 2, 265-305 MR.1224080 (94k:58055)

[ASvM] Adler, M.; Shiota, T.; van Moerbeke, P., "Pfaff $\tau$-functions", Math. Ann. 322 (2002), no. 3, pp. 423-476 MR1895702 (2003h:37136)

[DJKM] Date, E.; Jimbo, M.; Kashiwara, M.; Miwa, T., "Transformation groups for soliton equations", Proc. RIMS Sympos. on Nonlinear Integral Systems, World Scientific, Singapore (1983), pp. 39-119 MR.725700 (86a:58093)

[D] Dubrovin, B. A.; "Theta functions and non-linear equations", Russian Math. Surveys 36 (1981), no. 2, pp. 11-92 MR616797(83i:35149)

[F1] Fay, J. D., "Bilinear identities for Theta Functions", Preprint 
[F2] Fay, J. D., "Theta functions on Riemann surfaces", Lecture Notes in Mathematics, Vol. 352. Springer-Verlag, Berlin-New York, 1973. MR0335789 (49:569)

[GMP] Gómez González, E.; Muñoz Porras, J. M.; Plaza Martín, F. J., "Prym varieties, curves with automorphisms and the Sato Grassmannnian”, Math. Ann. 327 (2003), no. 4, pp. 609-639 MR2023311 (2004m:14053)

[GK] Grushevsky, S.; Krichever, I. M., "Integrable discrete Schrödinger equations and a characterization of Prym varieties by a pair of quadrisecants", arXiv:0705.2829

[KvdL] Kac, V. G.; van de Leur, J. W., "The n-component KP hierarchy and representation theory. Integrability, topological solitons and beyond", J. Math. Phys. 44 (2003), no. 8, 3245-3293. MR2006751 (2004g:37098)

[K1] Krichever, I.M., "Methods of algebraic geometry in the theory of non-linear equations", Russian Math. Surveys 32:6 (1977), 185-213

[K2] Krichever, I. M., "Integration of nonlinear equations by the methods of algebraic geometry", Functional Analysis and its Applications 11 (1977), no. 1, pp. 12-26 MR0494262 $(58: 13168)$

[K3] Krichever, I. M.; "Characterizing Jacobians via trisecants of the Kummer Variety", to appear in Annals in Mathematics, arXiv:math/0605625

[LM] Li, Y.; Mulase, M., "Category of morphisms of algebraic curves and a characterization of Prym varieties", Comm. Anal. Geom. 5 (1997), no. 2, 279-332

[Ma] Maffei, A., "The multicomponent KP and Fay trisecant formula", Internat. Math. Res. Notices 1996, no. 16, 769-791 MR1413872 (97g:14028)

[Mu1] Mulase, M., "Cohomological structure in soliton equations and Jacobian varieties", J. Differential Geom. 19 (1984), pp. 403-430. MR755232 (86f:14016)

[Mu2] Mulase, M., "A correspondence between an infinite Grassmannian and arbitrary vector bundles on algebraic curves", Proc. Symp. Pure Math. 49 Series A (1989), pp. 39-50. MR:1013124 (91f:14028)

[MP1] Muñoz Porras, J.M.; Plaza Martín, F.J., "Equations of the moduli space of pointed curves in the infinite Grassmannian", J. Differ. Geom. 51 (1999), pp. 431-469. MR:1726736 (2001a:14032)

[MP2] Muñoz Porras, J. M.; Plaza Martín, F. J., "Equations of Hurwitz schemes in the infinite Grassmannian”, Math. Nachr. 281 (2008), no. 7, 989-1012. MR2431573 (2009g:14036)

[SS] Sato, M.; Sato, Y., "Soliton equations as dynamical systems on infinite Grassman manifold", Lecture Notes Numer. Appl. Anal. 5 (1982), 259-271. MR730247 (86m:58072)

[Sh] Shiota, T., "Characterization of Jacobian varieties in terms of soliton equations", Invent. Math. 83 (1986), 333-382. MR818357(87j:14047)

[Sh2] Shiota, T., "Prym varieties and soliton equations", Infinite-dimensional Lie algebras and groups, Adv. Ser. Math. Phys., 7 (Luminy-Marseille, 1988), pp. 407-448, World Sci. Publishing, Teaneck, NJ, 1989. MR1026961 (91c:14038)

[SW] Segal, G.; Wilson, G., "Loop groups and equations of KdV type", Publ. Math. I.H.E.S. 61 (1985), 5-64. MR783348(87b:58039)

Departamento de Matemáticas, Universidad de Salamanca, Plaza de la Merced 1-4, 37008 Salamanca, Spain

E-mail address: esteban@usal.es

Departamento de Matemáticas, Universidad de Salamanca, Plaza de la Merced 1-4, 37008 Salamanca, Spain

E-mail address: jmp@usal.es

Departamento de Matemáticas, Universidad de Salamanca, Plaza de la Merced 1-4, 37008 Salamanca, Spain

E-mail address: fplaza@usal.es

Departamento de Matemáticas, Pontificia Universidad Católica de Chile, Casilla 306-22, Santiago, Chile

E-mail address: rubi@mat.puc.cl 